Usha Shankar, Neminath B. Naduvinamani*, and Hussain Basha

\title{
A generalized perspective of Fourier and Fick's laws: Magnetized effects of Cattaneo-Christov models on transient nan ofluid flow between two parallel plates with Brownian motion and thermophoresis
}

https://doi.org/10.1515/nleng-2020-0009

Received Jul 3, 2019; accepted Dec 28, 2019.

\begin{abstract}
Present research article reports the magnetized impacts of Cattaneo-Christov double diffusion models on heat and mass transfer behaviour of viscous incompressible, time-dependent, two-dimensional Casson nanofluid flow through the channel with Joule heating and viscous dissipation effects numerically. The classical transport models such as Fourier and Fick's laws of heat and mass diffusions are generalized in terms of Cattaneo-Christov double diffusion models by accounting the thermal and concentration relaxation times. The present physical problem is examined in the presence of Lorentz forces to investigate the effects of magnetic field on double diffusion process along with Joule heating. The non-Newtonian Casson nanofluid flow between two parallel plates gives the system of time-dependent, highly nonlinear, coupled partial differential equations and is solved by utilizing RK-SM and bvp4c schemes. Present results show that, the temperature and concentration distributions are fewer in case of Cattaneo-Christov heat and mass flux models when compared to the Fourier's and Fick's laws of heat and mass diffusions. The concentration field is a diminishing function of thermophoresis parameter and it is an increasing function of Brownian motion parameter. Finally, an excellent comparison between the present solutions and previously published results show the accuracy of the results and methods used to achieve the objective of the present work.
\end{abstract}

Keywords: Cattaneo-Christov heat and mass flux models; Joule heating; MHD flow

Usha Shankar, Department of Mathematics, Gulbarga University, Kalaburagi-585 106, Karnataka, India

Department of Karnataka Power Corporation Limited, Raichur Thermal Power Station, Shaktinagar, Raichur-584 170, India

\section{Introduction}

In most of the engineering systems, the process of heat transfer is suppressed due to the use of low thermal conductivity fluids and these fluids behaves like obstacles to magnify the thermal energy flow in the engineering systems. Therefore, to overcome these troubles in heat transfer process, it is very momentous to use the fluids which have larger thermal conductivity than the conventional fluids. Thus, scientists and researchers named that special class of fluids as "nanofluids". It is obvious that, nanofluids have higher thermal conductivity when compared to the common pure liquids. However, with the tremendous improvement and modification in the modern nanofluid technology, the nano-materials are adequately used in most of the scientific and bio-engineering applications such as probing of DNA structure, tumour obliteration via heating, MRI contrast enrichment, bio detection of pathogens, proteins detection, tissue engineering and so on [1-3]. Further, the nanofluid technology is used in lubrication systems, transportation, industries, drug delivery, defense, medicine, cosmetics, catalysis, food packing, automobiles and many other [4-6].

Nanofluids are the compounds of base fluids and nanoparticles with the mean sizes of the range $1-100 \mathrm{~nm}$. To intensify the thermal conductivity of the fluids, the working fluids are transformed to nanofluids, owing to their strong thermal conductivity than prevalent fluids in the given medium. Usually, the suspension of nano-materials includes the nanoparticles volume fraction (commonly < $5 \%$ ) of metal and which causes the magnification of ther-

\footnotetext{
*Corresponding Author: Neminath B. Naduvinamani, Department of Mathematics, Gulbarga University, Kalaburagi-585 106, Karnataka, India, E-mail: naduvinamaninb@yahoo.co.in

Hussain Basha, Department of Mathematics, Central University of Karnataka, Kalaburagi-585 367, India
} 
mal conductivity of nanomaterials. This fact was proved by Choi and Eastman [7]. With this idea of nanofluids, many of the researchers studied the heat and mass transfer process in various geometries by considering water as base fluid of Newtonian or viscous type in large scale. However, when the fluids are deformed between two boundaries approaching to each other or moving away from one another, such behaviour of fluid flows is named as squeezing flow. Generally, squeezing flows are produced by the application of normal stress to the moving plates/surfaces. Further, squeezing flows have number of applications in the field of engineering, industry and biomedicine. Particularly, in lubrication systems, polymer processing, flow of blood in vessels owing to the blood pressure, foam formation, food processing, hydro-dynamical machines, cooling towers, chemical processing, and bi-axial expansion of bubble boundaries, heating/cooling processes, injection moulding, compression, moisture migration, dampers and so on. Also, a few of other squeezing flows examples comprise the diarthrodial joints and valves related to the field of mathematical biology and bioengineering [8]. In many of the extreme operating systems the occurrence of unpredictable deviation of lubrication viscosity with respect to temperature in squeezing flow can be controlled by applying the external uniform magnetic field.

Following the pioneering work of Stefan [9], the thermal flow behaviour of viscous fluids in squeezing flow can be easily analyzed. However, for the first time Stefan [9] made an attempt to demonstrate the thermodynamic characteristic properties of squeezed flows in lubrication systems. Further, Stefan developed squeezed flow model by utilizing lubrication assumptions. Additionally, Stefan's work was continued by Reynolds [10] to study the behaviour of fluid flow between two elliptic plates. Further, a similar type problem of fluid flow through the rectangular channel was investigated by Archibald [11]. Leider and Bird [12] theoretically discussed the squeezed phenomena by considering the power-law liquid flow between two parallel disks. Their study reports that, the stress overshoot situation should be adequately explained via suitable rheological model to understand the fast squeezing phenomena. Domairry and Aziz [13] investigated the similar problem of viscous incompressible squeezing flow between two parallel disks in which they presented the influence of inertia, magnetic field on injection/suction process. Also, their results show that, the component of axial velocity amplified for the enhancing similarity variable in suction \& injection cases. Further, Siddiqui et al. [14] used the homotopy perturbation method to investigate the flow behaviour of magneto-hydrodynamic, two- dimensional squeezing flow of viscous fluid between two parallel plates. Their investigation disclosed that, the longitudinal velocity diminished for the increasing values of magnetic parameter.

The time-dependent, two-dimensional axisymmetric squeezing flow of viscous fluids between two parallel plates was analytically discussed by Rashidi et al. [15] by using analytical technique. Their discussion portrayed that, the squeezing parameter behaves like a function of flow velocity. Further, the heat transfer characteristics of viscoelastic fluid flow between two plates were reported by Kaushik et al. [16]. Their investigation reports that, the velocity of viscoelastic fluid diminishes in the transient state due to the presence of strong elastic behaviour. The unsteady magneto-hydrodynamic electrically conducting Jeffrey fluid flow between two parallel plates was analyzed by Muhammad et al. [17]. Their study states that, the magnifying Deborah number enhances the flow field in the channel. The analytical solutions for the magnetohydrodynamic incompressible axisymmetric flow between two infinite parallel disks via homotopy analysis method were discussed by Joneidi et al. [18]. Clearly their investigation remarked that, flow velocity shows the cross flow behaviour for enhancing magnetic field parameter at central portion of the channel. Hayat et al. [19] discussed the impact of magnetic field on the squeezed flow of second order fluid between two parallel disks via homotopy analysis method. It is noticed from their investigation that, the velocity field has opposite behaviour in suction and blowing cases. Duwairi et al. [20] analyzed the influence of squeezing parameter on heat transfer behaviour of viscous incompressible fluid flow through channel with viscous dissipation impact. However, their demonstration predicts that magnifying squeezing parameter diminish the heat transfer rate and increases the skin-friction coefficient and rising extrusion parameter diminishes the Nusselt number and increases the momentum transport coefficient in the flow region.

The process of simultaneous heat and mass transfer of viscous fluid between two parallel plates with squeezing effect was investigated by Mustafa et al. [21]. Their analytical investigation reports that, the magnitude of Nusselt number amplifies with rising Eckert and Prandtl numbers. Further, their study shows that, the concentration distribution is suppressed for destructive chemical reaction and magnified for the constructive chemical reaction parameters. Khan et al. [22] discussed the two-dimensional incompressible magnetized squeezed flow of viscous Casson fluid through the channel with porous medium impacts. However, their analysis shows that, the component of normal velocity diminished for the magnifying squeez- 
ing parameter values. Similar study was continued by Khan et al. [23] by considering the long infinite parallel plates via variation of parameters method. The characteristics properties and heat transfer behaviour of incompressible nanofluid flow between two non-parallel stretching walls was studied by Dogonchi and Ganji [24] via Duan-Rach method. Their investigation shows that Nusselt number, thermal field and viscosity of the fluid intensified with increment in stretching parameter. Also, their results shows that, for the cases of convergent and divergent channels, the temperature field rises with enhancement in heat generation parameter and further temperature field diminished for the magnifying radiation parameter values in the flow region. Dogonchi and Ganji [25] analytically investigated the double diffusion flow characteristics of nanofluid flow between two non-parallel walls with Joule heating impact by using Duan-Rach approach. Further, the behaviour of nanofluid was studied in terms of Brownian motion and thermophoresis effects with important mechanism. It is observed from their investigation that, the rate of local thermal energy transfer rises with upsurge in Schmidt number and concentration distribution upsurges with magnifying Brownian motion parameter. Further, Dogonchi and Ganji [25] demonstrated the influences of MHD on the time-dependent squeezing flow of nanofluid between two parallel plates by considering the impact of thermal radiation. Also, their study includes the dissipation effects to account the heat dissipation in the flow region. It is remarked from their investigation that, the thermal field and local heat transfer rate upsurges with increment in thermal radiation parameter. However, some of the pioneering efforts made in this direction to analyze the diffusion characteristics of nanofluids are [26-33].

The phenomena of combined heat and mass transfer has plenty of applications in many of the scientific and industrial processes, like refrigeration, heat exchangers, power collector equipment, air conditioners, crops damage, food processing and many others. In many of the physical problems, the process of heat transfer from one object to another object or in the same body occurs because of the temperature variation. Having this physical situation in account, Fourier's introduced his renowned law, referred as Fourier's law of heat conduction [34]. It is clear from the literature that, the Fourier's law is insufficient to predict the heat transfer behaviour completely. This is due to the occurrence initial disturbance instantly in all parts of the whole medium or object. In practical, there is no such material or object which obeys the Fourier's law. Therefore, to solve this problem Cattaneo [35] introduced the time relaxation factor so-called thermal inertia in conventional Fourier's law.
This relaxation factor indicates the resistive behaviour of the medium to change produced by the thermal gradient which is imposed externally to the medium, thus, this situation causes the thermal time delay in the medium. However, this amendment states that, the process of heat transfer is analogous to the propagation of thermal wave with confined speed in the medium. Further, Fourier's heat equation is parabolic in nature. Whereas, Cattaneo's heat transfer equation is governed by the hyperbolic equation. Christov [36] further improved the Cattaneo theory by introducing the time derivatives in Cattaneo's theory with Oldroyd upper convective derivatives that conserved the physical-invariant modeling. Further, the modified Cattaneo-Christov theory is subjected to stability along with uniqueness analysis by Ciarletta and Straughan [37]. Following are the some of the pioneering efforts made by the researchers to investigate the effects of CattaneoChristov double diffusion models on heat and mass transfer characteristics of viscous and non-viscous fluids.

The impacts of Cattaneo-Christov heat flux model on thermal convection in horizontal layer of incompressible Newtonian fluid was studied by Straughan [38] using D2 Chebyshev tau numerical method. However, literature [38] reports that, the easier occurrence of thermal convection takes place for the amplifying Cattaneo parameter. Hayat et al. [39] studied the impacts of Cattaneo-Christov heat flux model on flow of upperconvected Maxwell fluid (UCM) over a sheet with analytical technique. Their study shows that, the thermal field is stronger in Fourier's model when compared to CattaneoChristov double diffusion models. The characteristics of homogeneous-heterogeneous chemical reactions in case of Oldroyd-B fluid flow over a stretching surface with Cattaneo-Christov double diffusion models under magnetic field was discussed by Hayat et al. [40]. Further, the detailed study on Cattaneo-Christov double diffusion models can be found in [41-44].

Dogonchi and Ganji [45] utilized the Duan-Rach approach to investigate the influence of generalized Fourier's heat diffusion model on nanofluid flow between two parallel plates with magnetic effects. Farooq et al. [46] discussed the impacts of Cattaneo-Christov double diffusion models in the squeezing flow of Newtonian fluid past a stretched sheet in the presence of Darcy porous medium. Muhammad et al. [47] reported the phenomena of solutal and thermal stratification in the squeezing flow of twodimensional viscous fluid about a stretching surface with generalized thermal and mass flux models via optimal homotopy method. Recently, Farooq et al. [48] described the effects of Cattaneo-Christov double diffusion models on the squeezing flow of unsteady, viscous Newtonian fluid 
between two parallel plates by considering Darcy porous material and time-dependent chemical reaction. Akmal et al. [49] discussed the influence of Cattaneo-Christov heat and mass flux model on Newtonian nanofluid flow between two parallel plates with entropy generation analysis and magnetic effects. Their discussion reports that, the entropy generation and temperature field decayed for the magnifying thermal relaxation time parameter.

However, the technological applications of squeezing flows as mentioned before motivated us to consider this particular flow problem. Thus, the main objective of this article is to address the combined effects of heat and mass transfer on magnetized squeezed flow of viscous Casson nanofluid flow between two parallel plates via generalized Cattaneo-Christov double diffusion models with timedependent chemical reaction effects. The novelty of the present numerical work is to explore the effects CattaneoChristov double diffusion models in energy and concentration equations in place of classical Fourier's and Fick's laws owing to their drawbacks as mentioned before. After the comprehensive literature survey, it is observed that, the physical problem considered in this paper is not yet reported in the literature. Thus, authors made an attempt to solve this physical problem governed by time-dependent coupled nonlinear equations using Runge-Kutta fourth order method with shooting scheme (RK-SM) and bvp4c techniques. Further, the impacts of different flow parameters involved in the system are discussed physically and shown graphically.

\section{Stress tensor of Casson fluid}

The thermodynamic characteristic properties of Casson fluid was first examined by Casson in the year 1959 [50]. From the literature [50] it is observed that, Casson fluid is a shear thinning fluid with infinite viscosity at zero shear rates and a zero viscosity at an infinite rate of shear. However, the following rheological equation of Casson fluid flow $[22,50]$ is used to construct the flow equations for the present problem.

$$
\tau_{i j}=\left\{\begin{array}{l}
2\left(\mu_{B}+\frac{P_{y}}{\sqrt{2 \pi}}\right) e_{i j}, \pi>\pi_{c} \\
2\left(\mu_{B}+\frac{P_{y}}{\sqrt{2 \pi_{c}}}\right) e_{i j}, \pi<\pi_{c}
\end{array}\right.
$$

In this model, the symbol $\pi=e_{i j} . e_{i j}$ denotes the product of deformation components, $e_{i j}$ denotes $(i, j)^{\text {th }}$ deformation rate component, critical value is indicated by $\pi_{c}$, $P_{y}$ and $\mu_{B}$ indicate the yield stress and viscosity. Here, yield stress is the minimum stress required to produce the flow and below which no flow occurs.

\subsection{Mathematical formulation of the problem}

The impacts of Cattaneo-Christov heat and mass flux models on incompressible squeezed flow of magnetized twodimensional time-dependent viscous Casson nanofluid between two parallel plates separated by a distance $\pm h(t)= \pm l(1-\alpha t)^{1 / 2}$ with Brownian motion and thermophoresis effects have been examined numerically. Owing to the motion of the upper plate with velocity $\left(\frac{-\alpha l}{2 \sqrt{1-\alpha t}}\right)$ at a distance $\pm l(1-\alpha t)^{1 / 2}$ towards or away from the lower stationary plate situated at $y=0$, fluid flow occurs. In this case the symbol "l" signifies the initial position (when time $t=0$ ). Squeezing phenomena occurs when $\alpha>0$ till plates reach $t=1 / \alpha$ and plates are separated when $\alpha<0$. The Cartesian coordinate system is used to study the mathematical behaviour of this physical situation and in which $x$-coordinate is aligned along the axial flow direction of the channel, while $y$-axis is taken normal to axial coordinate. Fluid flow is regulated by applying the $B_{o}(1-\alpha t)^{-1 / 2}$ perpendicular to plates. Further, the squeezing rate parameter $\alpha$ has the dimension of (time) $)^{-1}$ and $\alpha t<1$. However, Figure 1(a) clearly illustrates the flow configuration with all necessary conditions and associated coordinate system of the present problem.

Further, Figure 1(b) identically demonstrates the impact of applied magnetic field on the flow behaviour before and after its application. Further, the Cattaneo-Christov double diffusion models are used to study the heat and mass flow behaviour of Casson nanofluid in a channel instead of conventional transport models. However, the thermal flux $q$ and mass flux $J$ accomplish the succeeding equations in terms of Cattaneo-Christov models [47, 48].

$$
\begin{aligned}
& q+\epsilon_{T}\left[\frac{\partial q}{\partial t}+V \cdot \nabla q+(\nabla \cdot V) q-q . \nabla V\right]=-\kappa \nabla T \\
& J+\epsilon_{C}\left[\frac{\partial J}{\partial t}+V \cdot \nabla J+(\nabla \cdot V) J-J . \nabla V\right]=-D_{B} \nabla C
\end{aligned}
$$

In the above Eqs. (2) and (3), heat flux is denoted by $q$ and $J$ represents the mass flux. Also, the symbols $\epsilon_{T}$ and $\epsilon_{C}$ describes the relaxation times with respect to temperature and concentration fields. $V$ is the velocity vector, the thermal conductivity of the fluid is denoted by $\kappa$ and $D_{B}$ be the coefficient of Brownian motion.

However, the classical Fourier's and Fick's laws can be sought back by imposing the condition $\epsilon_{T}=\epsilon_{C}=0$ on the Eqs. (2) and (3), respectively. Further, by applying the law of conservation mass condition, $\nabla \cdot V=0$, the Eqs. (2) and 


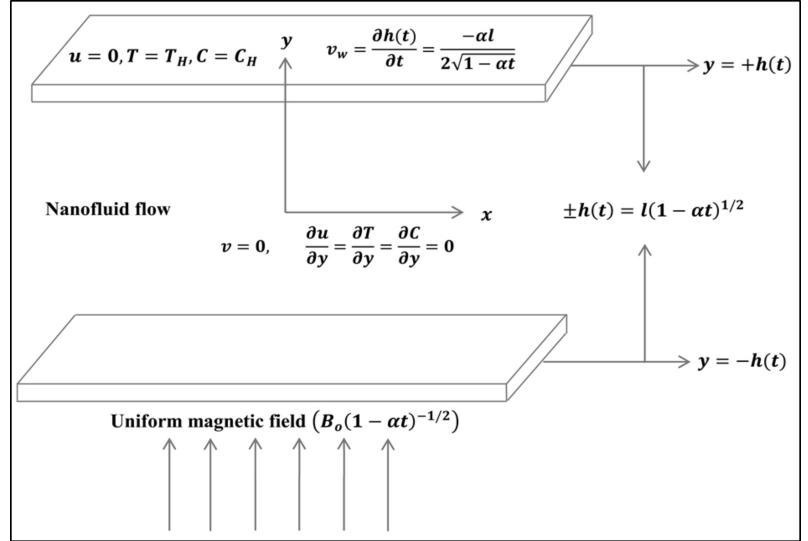

(a)

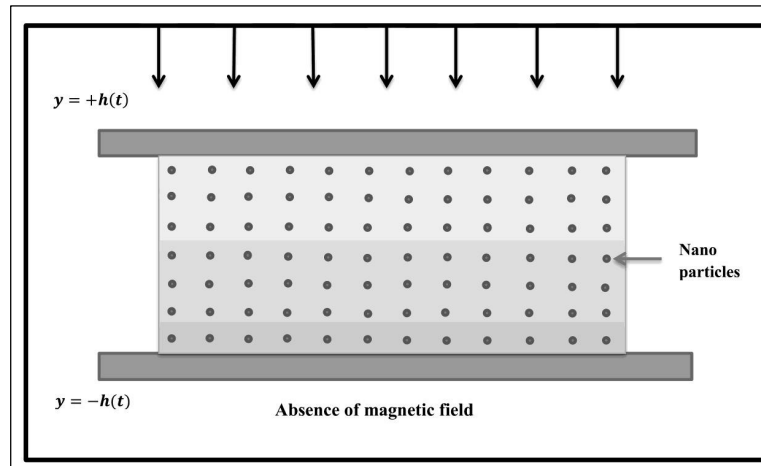

Flow configuration before squeezing.

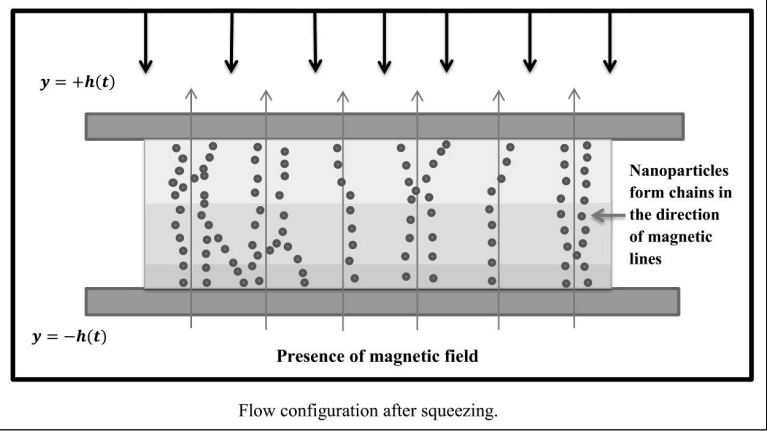

(b)

Figure 1: (a) Physical model and coordinate system of the investigated problem. (b) Influence of magnetic field on nanofluid flow behaviour

(3) transformed to the following form [47, 48].

$$
\begin{array}{r}
q+\epsilon_{T}\left[\frac{\partial q}{\partial t}+V . \nabla q-q . \nabla V\right]=-\kappa_{n f} \nabla T \\
J+\epsilon_{C}\left[\frac{\partial J}{\partial t}+V . \nabla J-J . \nabla V\right]=-D_{B} \nabla C
\end{array}
$$

Additionally, the heat dissipation owing to frictional forces generated through shear stress is accounted. Also, magnetic field and Joule heating effects are accounted respectively, in the momentum and energy equations. Further, a homogeneous chemical reaction of first order is assumed to be occurring. With these assumptions and by making the use of Casson fluid flow model described in section 2, the governing equations of transient squeezing flow of Casson nanofluid between two parallel plates with necessary conditions are obtained as follows.

\section{Law of conservation of mass:}

$$
\frac{\partial u}{\partial x}+\frac{\partial v}{\partial y}=0
$$

\section{Momentum diffusion equations:}

$$
\begin{gathered}
\frac{\partial u}{\partial t}+u \frac{\partial u}{\partial x}+v \frac{\partial u}{\partial y}=-\frac{1}{\rho_{n f}}\left(\frac{\partial p}{\partial x}\right) \\
+v_{n f}\left(1+\frac{1}{\beta}\right)\left(2 \frac{\partial^{2} u}{\partial x^{2}}+\frac{\partial^{2} u}{\partial y^{2}}+\frac{\partial^{2} v}{\partial y \partial x}\right)-\frac{\sigma B_{o}^{2}}{\rho_{n f}(1-\alpha t)} u \\
\frac{\partial v}{\partial t}+u \frac{\partial v}{\partial x}+v \frac{\partial v}{\partial y}=-\frac{1}{\rho_{n f}}\left(\frac{\partial p}{\partial y}\right) \\
+v_{n f}\left(1+\frac{1}{\beta}\right)\left(2 \frac{\partial^{2} v}{\partial x^{2}}+\frac{\partial^{2} v}{\partial y^{2}}+\frac{\partial^{2} u}{\partial y \partial x}\right)
\end{gathered}
$$

\section{Energy diffusion equation:}

$$
\begin{aligned}
& \frac{\partial T}{\partial t}+u \frac{\partial T}{\partial x}+v \frac{\partial T}{\partial y}+\epsilon_{T} \Sigma_{T}=\frac{\kappa_{n f}}{\left(\rho c_{p}\right)_{f}}\left(\frac{\partial^{2} T}{\partial x^{2}}+\frac{\partial^{2} T}{\partial y^{2}}\right) \\
& +\frac{\mu_{n f}}{\left(\rho c_{p}\right)_{f}}\left(1+\frac{1}{\beta}\right)\left(2\left(\frac{\partial u}{\partial x}\right)^{2}+\left(\frac{\partial u}{\partial y}+\frac{\partial v}{\partial x}\right)^{2}+2\left(\frac{\partial v}{\partial y}\right)^{2}\right) \\
& +\frac{\sigma B_{o}^{2}}{\left(\rho c_{p}\right)_{f}(1-\alpha t)} u^{2}+\frac{\left(\rho c_{p}\right)_{p}}{\left(\rho c_{p}\right)_{f}}\left[D_{B}\left\{\frac{\partial C}{\partial x} \frac{\partial T}{\partial x}+\frac{\partial C}{\partial y} \frac{\partial T}{\partial y}\right\}\right. \\
& \left.+\left(\frac{D_{T}}{T_{m}}\right)\left\{\left(\frac{\partial T}{\partial x}\right)^{2}+\left(\frac{\partial T}{\partial y}\right)^{2}\right\}\right]
\end{aligned}
$$

\section{Mass diffusion equation:}

$$
\begin{aligned}
& \frac{\partial C}{\partial t}+u \frac{\partial C}{\partial x}+v \frac{\partial C}{\partial y}+\epsilon_{C} \Sigma_{C}=D_{B}\left(\frac{\partial^{2} C}{\partial x^{2}}+\frac{\partial^{2} C}{\partial y^{2}}\right) \\
& -\frac{k_{1}}{(1-\alpha t)} C+\left(\frac{D_{T}}{T_{m}}\right)\left(\frac{\partial^{2} T}{\partial x^{2}}+\frac{\partial^{2} T}{\partial y^{2}}\right)
\end{aligned}
$$

In the above Eqs. (6)-=(10), $u$ and $v$ are the axial and radial velocities along $x$ and $y$ axis, $\rho_{n f}$ is nanofluid density, $v_{n f}$ depicts kinematic viscosity of nanofluid, $\mu_{n f}$ is the dynamic viscosity nanofluid, $p$ is the pressure, $T$ and $C$ are the nanofluid temperature and concentration, $\beta$ denotes the Casson fluid parameter, $D_{B}$ is the coefficient of Brownian motion, $D_{T}$ is the thermophoresis diffusion coefficient, $\sigma$ describes the electrical conductivity, $B_{o}$ magnetic field, $\kappa$ denotes the thermal conductivity, $\alpha$ is squeezing rate, $T_{H}, C_{H}$ describes temperature and concentration of the 
upper plate and $k_{1}$ is the time-dependent chemical reaction coefficient

Further, the values of $\Sigma_{T}$ and $\Sigma_{C}$ in the Eqs. (9) and (10) are examined as follows:

$$
\begin{aligned}
& \Sigma_{T}=\frac{\partial^{2} T}{\partial t^{2}}+\frac{\partial u}{\partial t} \frac{\partial T}{\partial x}+2 u \frac{\partial^{2} T}{\partial t \partial x}+2 v \frac{\partial^{2} T}{\partial t \partial y}+\frac{\partial v}{\partial t} \frac{\partial T}{\partial y} \\
& +u \frac{\partial u}{\partial x} \frac{\partial T}{\partial x}+v \frac{\partial v}{\partial y} \frac{\partial T}{\partial y}+u \frac{\partial v}{\partial x} \frac{\partial T}{\partial y}+v \frac{\partial u}{\partial y} \frac{\partial T}{\partial x}+2 u v \frac{\partial^{2} T}{\partial x \partial y} \\
& +u^{2} \frac{\partial^{2} T}{\partial x^{2}}+v^{2} \frac{\partial^{2} T}{\partial y^{2}} \\
& \Sigma_{C}=\frac{\partial^{2} C}{\partial t^{2}}+\frac{\partial u}{\partial t} \frac{\partial C}{\partial x}+2 u \frac{\partial^{2} C}{\partial t \partial x}+2 v \frac{\partial^{2} C}{\partial t \partial y}+\frac{\partial v}{\partial t} \frac{\partial C}{\partial y} \\
& +u \frac{\partial u}{\partial x} \frac{\partial C}{\partial x}+v \frac{\partial v}{\partial y} \frac{\partial C}{\partial y}+u \frac{\partial v}{\partial x} \frac{\partial C}{\partial y}+v \frac{\partial u}{\partial y} \frac{\partial C}{\partial x}+2 u v \frac{\partial^{2} C}{\partial x \partial y} \\
& +u^{2} \frac{\partial^{2} C}{\partial x^{2}}+v^{2} \frac{\partial^{2} C}{\partial y^{2}}
\end{aligned}
$$

Thus, the required dimensional temperature and concentration equations are obtained by replacing the examined values of $\Sigma_{T}$ and $\Sigma_{C}$ in the corresponding energy and concentration equations. In view of this reason, the Eqs. (11) and (12) are replaced in the Eqs. (9) and (10) to the obtained modified heat and mass transport equations, thus, we have $[47,48]$.

$$
\left.\begin{array}{l}
\frac{\partial T}{\partial t}+u \frac{\partial T}{\partial x}+v \frac{\partial T}{\partial y}+\epsilon_{T}\left(\frac{\partial^{2} T}{\partial t^{2}}+\frac{\partial u}{\partial t} \frac{\partial T}{\partial x}+2 u \frac{\partial^{2} T}{\partial t \partial x}\right. \\
+2 v \frac{\partial^{2} T}{\partial t \partial y}+\frac{\partial v}{\partial t} \frac{\partial T}{\partial y}+u \frac{\partial u}{\partial x} \frac{\partial T}{\partial x}+v \frac{\partial v}{\partial y} \frac{\partial T}{\partial y}+u \frac{\partial v}{\partial x} \frac{\partial T}{\partial y} \\
\left.+v \frac{\partial u}{\partial y} \frac{\partial T}{\partial x}+2 u v \frac{\partial^{2} T}{\partial x \partial y}+u^{2} \frac{\partial^{2} T}{\partial x^{2}}+v^{2} \frac{\partial^{2} T}{\partial y^{2}}\right) \\
=\frac{k_{n f}}{\left(\rho c_{p}\right)_{f}}\left(\frac{\partial^{2} T}{\partial x^{2}}+\frac{\partial^{2} T}{\partial y^{2}}\right)+\frac{\mu_{n f}}{\left(\rho c_{p}\right)_{f}} \\
\left(1+\frac{1}{\beta}\right)\left(2\left(\frac{\partial u}{\partial x}\right)^{2}+\left(\frac{\partial u}{\partial y}+\frac{\partial v}{\partial x}\right)^{2}+2\left(\frac{\partial v}{\partial y}\right)^{2}\right) \\
+\frac{\sigma B_{o}^{2}}{\left(\rho c_{p}\right)_{f}(1-\alpha t)} u^{2}+\frac{\left(\rho c_{p}\right)_{p}}{\left(\rho c_{p}\right)_{f}}\left[D_{B}\left\{\frac{\partial C}{\partial x} \frac{\partial T}{\partial x}+\frac{\partial C}{\partial y} \frac{\partial T}{\partial y}\right\}\right. \\
\left.+\left(\frac{D_{T}}{T_{m}}\right)\left\{\left(\frac{\partial T}{\partial x}\right)^{2}+\left(\frac{\partial T}{\partial y}\right)^{2}\right\}\right] \\
\frac{\partial C}{\partial t}+u \frac{\partial C}{\partial x}+v \frac{\partial C}{\partial y}+\epsilon_{C}\left(\frac{\partial^{2} C}{\partial t^{2}}+\frac{\partial u}{\partial t} \frac{\partial C}{\partial x}+2 u \frac{\partial^{2} C}{\partial t \partial x}\right. \\
+2 v \frac{\partial^{2} C}{\partial t \partial y}+\frac{\partial v}{\partial t} \frac{\partial C}{\partial y}+u \frac{\partial u}{\partial x} \frac{\partial C}{\partial x}+v \frac{\partial v}{\partial y} \frac{\partial C}{\partial y}+u \frac{\partial v}{\partial x} \frac{\partial C}{\partial y} \\
\left.+v \frac{\partial u}{\partial y} \frac{\partial C}{\partial x}+2 u v \frac{\partial^{2} C}{\partial x \partial y}+u^{2} \frac{\partial^{2} C}{\partial x^{2}}+v^{2} \frac{\partial^{2} C}{\partial y^{2}}\right)= \\
D_{B}\left(\frac{\partial^{2} C}{\partial x^{2}}+\frac{\partial^{2} C}{\partial y^{2}}\right)-\frac{k_{1}}{(1-\alpha t)} C+\left(\frac{D_{T}}{T_{m}}\right)\left(\frac{\partial^{2} T}{\partial x^{2}}+\frac{\partial^{2} T}{\partial y^{2}}\right)
\end{array}\right\}
$$

The associated boundary conditions for the timedependent squeezing flow are as follows:

$$
u=0, \quad v=v_{w}=\frac{d h}{d t}, \quad T=T_{H}, \quad C=C_{H}, \quad \text { at } y=h(t)
$$

$$
\frac{\partial u}{\partial y}=0, \quad v=0, \frac{\partial T}{\partial y}=0, \frac{\partial C}{\partial y}=0 \text { at } y=0
$$

In the Eq. 15(a), $v_{w}=\frac{d h}{d t}$ indicate the velocity with which upper plate is moving towards or away from the lower plate which is kept at a distance $y=0$ from the upper plate at $y=h(t)$. Further, the value of the $v_{w}$ is examined as $\left(\frac{-\alpha l}{2 \sqrt{1-\alpha t}}\right)$.

\subsection{Similarity transformation approach}

The physical problem discussed in this paper is governed by the time-dependent, coupled nonlinear partial differential equations (PDEs) of higher order. Owing to the complexity of governing equations, the analytical or direct methods are insufficient to solve these PDEs completely. However, to tackle this problem, the complex PDEs are first converted to the group of nonlinear ordinary differential equations (ODEs) by utilizing the applicable transformations. Further, the reduced nonlinear ODEs are solved by employing RK-SM [51] and bvp4c [52] methods. Thus, following suitable similarity transformations [21] are used to this end.

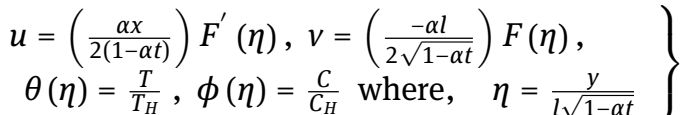

The pressure gradient term in the Eqs. (7) and (8) is eliminated by using the Eqs. (6) and (16) and the resulting momentum equation is of the following form:

$$
\left.\begin{array}{l}
\left(1+\frac{1}{\beta}\right) F^{\prime \prime \prime \prime}(\eta)-S\left(\eta F^{\prime \prime \prime}(\eta)+3 F^{\prime \prime}(\eta)+F^{\prime}(\eta) F^{\prime \prime}(\eta)\right. \\
\left.-F(\eta) F^{\prime \prime \prime}(\eta)\right)-H a^{2} F^{\prime \prime}(\eta)=0
\end{array}\right\}
$$

Further, the thermal and concentration diffusion equations are articulated in terms of similarity variable. To this end, using Eq. (16) into the Eqs. (13) and (14), the transformed temperature and concentration equations are as follows:

$$
\left.\begin{array}{l}
\theta^{\prime \prime}(\eta)+\operatorname{PrEc}\left(\left(1+\frac{1}{\beta}\right)\left(F^{\prime \prime 2}(\eta)+4 \delta^{2} F^{\prime 2}(\eta)\right)\right. \\
\left.+H a^{2} F^{\prime 2}(\eta)\right)+\operatorname{PrS}\left(F(\eta) \theta^{\prime}(\eta)-\eta \theta^{\prime}(\eta)\right) \\
+N_{b} \theta^{\prime}(\eta) \phi^{\prime}(\eta)+N_{t}\left(\theta^{\prime}(\eta)\right)^{2}-\operatorname{Pr} S \Omega_{T}\left(\eta^{2} \theta^{\prime \prime}(\eta)\right. \\
+3 \eta \theta^{\prime}(\eta)-2 \eta F(\eta) \theta^{\prime \prime}(\eta)-3 F(\eta) \theta^{\prime}(\eta) \\
\left.-\eta F^{\prime}(\eta) \theta^{\prime}(\eta)+F(\eta) F^{\prime}(\eta) \theta^{\prime}(\eta)+F^{2}(\eta) \theta^{\prime \prime}(\eta)\right)=0
\end{array}\right\}
$$

$$
\begin{aligned}
& \phi^{\prime \prime}(\eta)+\operatorname{Sc} S\left(F(\eta) \phi^{\prime}(\eta)-\eta \phi^{\prime}(\eta)\right)-S c K r \phi(\eta) \\
& -S c S \Omega_{C}\left(\eta^{2} \phi^{\prime \prime}(\eta)+3 \eta \phi^{\prime}(\eta)-2 \eta F(\eta) \phi^{\prime \prime}(\eta)\right. \\
& -3 F(\eta) \phi^{\prime}(\eta)-\eta F^{\prime}(\eta) \phi^{\prime}(\eta)+F(\eta) F^{\prime}(\eta) \phi^{\prime}(\eta) \\
& \left.+F^{2}(\eta) \phi^{\prime \prime}(\eta)\right)+\left(\frac{N_{b}}{N_{t}}\right) \theta^{\prime \prime}(\eta)=0
\end{aligned}
$$

Similarly, the relevant boundary conditions are reduced to dimensionless form by replacing the Eq. (16) into the Eqs. 15(a) and 15(b), as follows.

$F(\eta)=0, \quad F^{\prime \prime}(\eta)=0, \quad \theta^{\prime}(\eta)=0, \quad \phi^{\prime}(\eta)=0$ at $\eta=0$ 
$F(\eta)=1, F^{\prime}(\eta)=0, \theta(\eta)=1, \phi(\eta)=1$ at $\eta=1$

(20b)

Further, the various dimensionless parameters in the Eqs. (17)-(19) are considered as follows:

$$
\Omega_{T}=\left(\frac{\alpha \epsilon_{T}}{2(1-\alpha t)}\right)
$$

(Thermal relaxation time parameter), $H a=B_{o} l \sqrt{\frac{\sigma}{\mu}}$ (Hartmann number), $\Omega_{C}=\left(\frac{\alpha \epsilon_{C}}{2(1-\alpha t)}\right)$ (Concentration relaxation time parameter), $S=\frac{\alpha l^{2}}{2 v}$ (Squeezing number), $N_{b}=$ $\left(\frac{\left(\rho c_{p}\right)_{p}}{\left(\rho c_{p}\right)_{f}}\right)\left(\frac{D_{B} C_{H}}{\left(\frac{K}{\rho c_{p}}\right)}\right)$ (Brownian motion parameter), Ec = $\frac{\alpha^{2} x^{2}}{4 C_{p} T_{H}(1-\alpha t)^{2}}$ (Eckert number), $N_{t}=\left(\frac{\left(\rho c_{p}\right)_{p}}{\left(\rho c_{p}\right)_{f}}\right)\left(\frac{D_{T} T_{H}}{T_{m}\left(\frac{k}{\rho c_{p}}\right)}\right)$ (Thermophoresis parameter), $\operatorname{Pr}=\frac{\mu C_{p}}{\kappa}$ (Prandtl number) $K r=\frac{k_{1} l^{2}}{v}$ (Time-dependent chemical reaction parameter) and $S c=\frac{v}{D}$ (Schmidt number).

Thus, the considered physical problem under the application of magnetic field is completely portrayed in terms of squeezing parameter $S$ and which in turn governed by the squeezing rate $\alpha$. The condition $S>0$ related to motion of plates away from each other and $S<0$ corresponds to the plates approaching towards each other, so-called squeezing process. Also, the effects of Cattaneo-Christov double diffusion models on flow behaviour are examined in terms of thermal and concentration relaxation time parameters. Further, the characteristics of nanofluids are depicted in terms of Brownian motion and thermophoresis parameters. Prandtl, Hartmann and Eckert numbers are utilized to control the flow and heat transfer process. Also, Schmidt number is used to describe the flows when there exist simultaneous heat and mass transfer phenomenon. Further, the homogenous chemical reaction of first order is illustrated in terms of time-dependent chemical reaction parameter.

\subsection{Physical quantities of engineering interest}

Physical quantities of engineering interest such as, momentum transport coefficient, heat and mass transfer rates for the present problem are defined as follows [21].

$$
\left.\begin{array}{l}
C_{f}=\frac{\mu_{n f}}{\rho_{n f}}\left(1+\frac{1}{\beta}\right)\left(\frac{\left(\frac{\partial u}{\partial y}\right)_{y=h(t)}}{v_{w}^{2}}\right) \\
N u=-l \kappa\left(\frac{\left(\frac{\partial T}{\partial y}\right)_{y=h(t)}}{\kappa T_{H}}\right) \\
S h=-l D_{B}\left(\frac{\left(\frac{\partial c}{\partial y}\right)_{y=h(t)}}{D_{B} C_{H}}\right)
\end{array}\right\}
$$

Thus, in terms of Eq. (16), the Eq. (21) can be expressed as follows:

$$
\left.\begin{array}{l}
\frac{l^{2}}{x^{2}(1-\alpha t)} \operatorname{Re}_{x} C_{f}=\left(1+\frac{1}{\beta}\right) F^{\prime \prime}(1) \\
\sqrt{(1-\alpha t)} N u=-\theta^{\prime}(1) \\
\sqrt{(1-\alpha t)} S h=-\phi^{\prime}(1)
\end{array}\right\}
$$

\section{Numerical solution procedure}

The channel flow of unsteady two-dimensional Casson nanofluid with Cattaneo-Christov heat and mass flux models with time-dependent chemical reaction and Joule dissipation is associated with highly nonlinear coupled flow equations. Due to the inadequacy of the analytical methods, the present problem is solved via numerical approaches namely, RK-SM and bvp4c method. Thus, the Eqs. (17)-(19) with Eq. (20) are solved using RK-SM and bvp4c techniques. However, the RK-SM method begins by discretising the coupled nonlinear higher order ODEs into the set of first order ODEs. Thus, following are the discretized first order ODEs used for numerical calculations.

$$
\begin{aligned}
& \frac{d F_{0}}{d \eta}=F_{1} \\
& \frac{d F_{1}}{d \eta}=F_{2} \\
& \frac{d F_{2}}{d \eta}=F_{3} \\
& \frac{d F_{3}}{d \eta}=\left(\frac{1}{1+\frac{1}{\beta}}\right)\left(S \left(\eta F_{3}(\eta)+3 F_{2}(\eta)+F_{1}(\eta) F_{2}(\eta)\right.\right. \\
& \left.\left.-F_{0}(\eta) F_{3}(\eta)\right)+H a^{2} F_{2}(\eta)\right)
\end{aligned}
$$

$$
\left.\frac{d \theta_{0}=\theta_{1}}{d \eta} \frac{d \theta_{1}}{d \eta}=\left(\frac{1}{1-H u}\right)\left(\begin{array}{l}
\operatorname{Pr} S\left(\eta \theta_{1}(\eta)-F_{0}(\eta) \theta_{1}(\eta)\right) \\
-\operatorname{Pr} E c\left(\left(1+\frac{1}{\beta}\right)\left(F_{2}^{2}(\eta)+4 \delta^{2} F_{1}^{2}(\eta)\right)\right. \\
\left.+H a^{2} F_{1}^{2}(\eta)\right)+S \operatorname{Sr} \Omega_{T}\left(3 \eta \theta_{1}(\eta)\right. \\
-3 F(\eta) \theta_{1}(\eta)-\eta F_{1}(\eta) \theta_{1}(\eta) \\
\left.+F_{0}(\eta) F_{1}(\eta) \theta_{1}(\eta)\right)-N_{b} \theta_{1}(\eta) \phi_{1}(\eta) \\
-N_{t}\left(\theta_{1}(\eta)\right)^{2}
\end{array}\right)\right\}
$$




$$
\begin{aligned}
& \frac{d \phi_{0}}{d \eta}=\phi_{1} \\
& \frac{d \phi_{1}}{d \eta}=\left(\frac{1}{1-U h}\right)\left(S S c \Omega _ { C } \left(3 \eta \phi_{1}(\eta)-3 F(\eta) \phi_{1}(\eta)\right.\right. \\
& \left.-\eta F_{1}(\eta) \phi_{1}(\eta)+F_{0}(\eta) F_{1}(\eta) \phi_{1}(\eta)\right)+S c S\left(\eta \phi_{1}(\eta)\right. \\
& \left.\left.-F_{0}(\eta) \phi_{1}(\eta)\right)+S c K r \phi_{0}(\eta)-\left(\frac{N_{b}}{N_{t}}\right) \frac{d \theta_{1}}{d \eta}\right)
\end{aligned}
$$

The values of $H u$ and $U h$ in the above Eqs. (24) and (25) are given as, $H u=\operatorname{SPr} \Omega_{T}\left(\eta^{2}-2 \eta F_{0}(\eta)+F_{0}^{2}(\eta)\right)$ and $U h=S S c \Omega_{C}\left(\eta^{2}-2 \eta F_{0}(\eta)+F_{0}^{2}(\eta)\right)$. Also, we have considered following boundary conditions to solve the Eqs. (23)-(25) with $F(\eta)=F_{0}(\eta), \theta(\eta)=\theta_{0}(\eta)$ and $\phi(\eta)=\phi_{0}(\eta)$.

$$
\left.\begin{array}{c}
F_{0}(\eta)=0, F_{2}(\eta)=0, \text { at } \eta=0 \\
F_{0}(\eta)=1, F_{1}(\eta)=0 \text { at } \eta=1
\end{array}\right\}
$$

Further, the current shooting technique initiates by converting boundary value problem (BVP) into an initial value problem (IVP) by predicting appropriate guesses of $F_{1}(0), F_{3}(0), \theta_{0}(0)$ and $\phi_{0}(0)$. Consequently, the converted IVP is solved by employing RK-SM. Further, in RKSM scheme, the convergence condition is governed by the suitable initial guesses of the unknown quantities. However, for the accurate similarity solutions over the nondimensional time $t$, the convergence criteria is fixed as $10^{-5}$ and $h=0.01$ be the step size. Further, once the convergence criteria is reached, the set of ODEs are integrated by using the RK-SM to obtain the required results.

\section{Physical interpretation of the obtained results}

\subsection{Validation of the present computer code}

The guarantee of used numerical scheme and obtained similarity solutions are verified by comparing with the previously published results of Wang [53], Khan et al. [54], Dogonchi and Ganji [45] and Mustafa et al. [21] for skinfriction coefficient and Nusselt number, respectively. Further, Table 1(a) clearly indicates the comparison of present results with the results of Wang [53], Khan et al. [54], and Table 1(b) shows the validation of current solutions with the solutions of Dogonchi and Ganji [45] and Table 1(c) portrays the comparison of present similarity solutions with Mustafa et al. [21]. However, it is observed from the Tables 1(a)-1(c) that, there exists an excellent agreement between the present numerical results and the results of Wang [53], Khan et al. [54], Dogonchi and Ganji [45] and Mustafa et al. [21] for various values of squeezing parameter. Additionally, Table 1(c) describes that, the magnitude of $\theta^{\prime}(1)$ enhanced for higher values of $E c$ and $P r$. Thus, from the Tables 1(a)-1(c), it is observed that, the similarity solutions obtained based on RK-SM and bvp4c techniques are highly guaranteed and reasonable.

\subsection{Comparative results between Runge-Kutta scheme and bvp $4 c$ matlab function}

The comparative results obtained based on Runge-Kutta scheme and bvp4c matlab function are illustrated in the Tables 2 and 3, respectively. The numerical values of $F(\eta)$, $\theta(\eta)$ and $\phi(\eta)$ calculated based on RK-SM is shown in the Table 2. Similarly, $F(\eta), \theta(\eta)$ and $\phi(\eta)$ values evaluated based on bvp4c technique is tabulated in the Table 3. The analysis of Tables 2 and 3 portrays that, there exists an excellent agreement between RK-SM and bvp4c methods. Thus, with this comparison it is remarked that, the numerical values obtained based on RK-SM and bvp4c are highly accurate and found to be reasonable. However, this comparison shows the accuracy of RK-SM and bvp4c methods for solving flow equations.

\subsection{Results and discussion:}

The impact of squeezing parameter $(S)$ on the flow behaviour is clearly described in this paragraph. However, Figures 2-5 describe the effect of $S$ on velocity field, temperature and concentration profiles, respectively. Figure 2 clearly portrays that, the radial velocity field diminished for $S>0$ and enhanced for $S<0$. Clearly, the quantity $S>0$ associated with the moment of the plates away from one another and $S<0$ is related to the moment of plates close to one another. However, the variation of velocity in the flow region is mainly owing to the reason that, for $S>0$ fluid sucked into the channel and hence velocity increases. On the other hand, for $S<0$ liquid released out from the channel and creates the liquid drop inside the channel and hence flow velocity diminished. Figure 3 shows that, for $S>0$, the axial velocity profile decreased in the region $0 \leq \eta \leq 0.45$ and increased in the remaining region of the 
Table 1a: Comparison of present similarity solutions with Wang [53] and Khan et al. [54] results for $F^{\prime \prime}$ (1) for different values of $S$ when $H a=N_{b}=N_{t}=\Omega_{T}=\Omega_{C}=0$

\begin{tabular}{ccccc}
\hline Squeezing number $(S)$ & Wang [53] & Khan et al. [54] & $\begin{array}{c}\text { Present results } \\
\text { (RK-SM) }\end{array}$ & Present results (bvp4c) \\
\hline-0.9780 & -2.235 & -2.1915 & -2.1915 & -2.1915 \\
-0.4977 & -2.6272 & -2.6193 & -2.6193 & -2.6193 \\
-0.09998 & -2.9279 & -2.9277 & -2.9277 & -2.9277 \\
0 & -3.000 & -3.000 & -3.000 & -3.000 \\
0.09403 & -3.0665 & -3.0663 & -3.0663 & -3.0663 \\
0.4341 & -3.2969 & -3.2943 & -3.2943 & -3.2943 \\
1.1224 & -3.714 & -3.708 & -3.708 & -3.708 \\
\hline
\end{tabular}

Table 1b: Comparison of present similarity solutions with Mustafa et al. [21] and Dogonchi and Ganji [45] results for $F^{\prime \prime}(1)$ and $\theta^{\prime}$ (1) for different values of $S$ when $H a=N_{b}=N_{t}=\Omega_{T}=\Omega_{C}=0$

\begin{tabular}{|c|c|c|c|c|c|c|c|c|}
\hline \multirow{2}{*}{$\begin{array}{l}\text { Squeezing } \\
\text { number } \\
S\end{array}$} & \multicolumn{2}{|c|}{ Mustafa et al. [21] } & \multicolumn{2}{|c|}{ Dogonchi and Ganji [45] } & \multicolumn{2}{|c|}{ Present results (RK-SM) } & \multicolumn{2}{|c|}{ Present results (bvp4c) } \\
\hline & $-F^{\prime \prime}(1)$ & $-\theta^{\prime}(1)$ & $-F^{\prime \prime}(1)$ & $-\theta^{\prime}(1)$ & $-F^{\prime \prime}(1)$ & $-\theta^{\prime}(1)$ & $-F^{\prime \prime}(1)$ & $-\theta^{\prime}(1)$ \\
\hline-1.0 & 2.170090 & 3.319899 & 2.170091 & 3.319888 & 2.170090 & 3.319899 & 2.170114 & 3.319912 \\
\hline-0.5 & 2.614038 & 3.129491 & 2.617403 & 3.129491 & 2.617403 & 3.129491 & 2.617417 & 3.129495 \\
\hline 0.01 & 3.007134 & 3.047092 & 3.007133 & 3.047091 & 3.007133 & 3.047091 & 3.007133 & 3.047092 \\
\hline 0.5 & 3.336449 & 3.026324 & 3.336449 & 3.026323 & 3.336449 & 3.026323 & 3.336444 & 3.026345 \\
\hline 2.0 & 4.167389 & 3.118551 & 4.167041 & 3.113386 & 4.167389 & 3.118550 & 4.167385 & 3.118641 \\
\hline
\end{tabular}

Table 1c: Comparison of present similarity solutions with the results of Mustafa et al. [21] for $-\theta^{\prime}(1)$ for various values of $P r$ and $E c$ when $H a=N_{b}=N_{t}=\Omega_{T}=\Omega_{C}=0$

\begin{tabular}{|c|c|c|c|c|}
\hline $\operatorname{Pr}$ & $E c$ & Mustafa et al. [21] & $\begin{array}{l}\text { Present results } \\
\text { (RK-SM) }\end{array}$ & $\begin{array}{l}\text { Present results } \\
\quad \text { (bvp4c) }\end{array}$ \\
\hline 0.5 & 1.0 & 1.522368 & 1.522368 & 1.522374 \\
\hline 1.0 & & 3.026324 & 3.026324 & 3.026345 \\
\hline 2.0 & & 5.980530 & 5.980530 & 5.980604 \\
\hline 5.0 & & 14.43941 & 14.43941 & 14.43955 \\
\hline \multirow[t]{4}{*}{1.0} & 0.5 & 1.513162 & 1.513162 & 1.513172 \\
\hline & 1.2 & 3.631588 & 3.631588 & 3.631615 \\
\hline & 2.0 & 6.052647 & 6.052647 & 6.052691 \\
\hline & 5.0 & 15.13162 & 15.13162 & 15.13172 \\
\hline
\end{tabular}

channel. Similarly, for $S<0$, the axial velocity field enhanced in region $0 \leq \eta \leq 0.45$ and decreased in region $0.45 \leq \eta \leq 1$. Further, from Figure 3 it is observed that, due to the variation in velocity at the boundaries cross flow behaviour is noticed at the central portion of channel. Furthermore, from Figure 4 it is remarked that, the temperature profile diminished for $S>0$ and it is enhanced for $S<0$. Practically, increasing squeezing parameter $(S>0)$ decreases the squeezing force and cause to decay the temperature profile in the flow region. Further, from Figure 4 it is noticed that, an increment in temperature profile is quite obvious for $S<0$, because, an increment in squeezing number closely associated with the decay of kinematic viscosity of the fluid, an increment in length between the plates, and an increment in speed with which the plates move. Further, Figure 5 portrays that, the concentration profile increased for $S>0$ and suppressed for $S<0$. However, the influence of $S$ on concentration field near the boundaries is insignificant when compared to the other portion of the channel and which clearly observed in Figure 5.

Figures 6-9 illustrates the effect of Casson fluid parameter $(\beta)$ on velocity, temperature and concentration profiles. From Figure 6 it is observed that, the radial velocity profile diminished for the increasing values of $\beta$. This variation in velocity field is mainly due to the fact that, $a$ small upsurge in $\beta$ results the enhancement in the fluid viscosity and hence this increase in viscosity offers more opposition to the fluid flow inside the channel. Therefore, the radial velocity profile decreased for the increasing $\beta$ values. Further, Figure 7 portrays the behaviour of axial flow and it is observed that, the axial velocity profile suppressed in the region $0 \leq \eta<0.48$ and increased in the remaining region of the channel for the increasing values of $\beta$. Further, it is clearly noticed from Figure 7 that, due to the variation of velocity near boundaries of the channel, a 
Table 2: Numerical values of $F(\eta), \theta(\eta)$ and $\phi(\eta)$ for different set of $S$, obtained based on RK-SM technique with fixed values of $H a=$ $0.1, \Omega_{T}=0.5, \Omega_{C}=1.5, N_{t}=N_{b}=0.1, E c=0.1, \beta=0.7, \operatorname{Pr}=0.7, K r=0.5$

\begin{tabular}{|c|c|c|c|c|}
\hline \multicolumn{5}{|c|}{ Results obtained based on RK-SM } \\
\hline$S$ & $\eta$ & $F(\eta)$ & $\theta(\eta)$ & $\phi(\eta)$ \\
\hline \multirow{5}{*}{-0.6} & 0.2 & 0.300430 & 1.144458 & 0.714478 \\
\hline & 0.4 & 0.574735 & 1.139841 & 0.734392 \\
\hline & 0.6 & 0.797794 & 1.122990 & 0.778070 \\
\hline & 0.8 & 0.946403 & 1.081340 & 0.860672 \\
\hline & 1.0 & 1.000000 & 1.000000 & 1.000000 \\
\hline \multirow{5}{*}{-0.4} & 0.2 & 0.298894 & 1.140245 & 0.723074 \\
\hline & 0.4 & 0.572404 & 1.135847 & 0.742846 \\
\hline & 0.6 & 0.795794 & 1.119779 & 0.785514 \\
\hline & 0.8 & 0.945577 & 1.079680 & 0.865306 \\
\hline & 1.0 & 1.000000 & 1.000000 & 1.000000 \\
\hline \multirow{5}{*}{-0.2} & 0.2 & 0.297412 & 1.136311 & 0.731141 \\
\hline & 0.4 & 0.570150 & 1.132118 & 0.750774 \\
\hline & 0.6 & 0.793855 & 1.116783 & 0.792478 \\
\hline & 0.8 & 0.944772 & 1.078138 & 0.869619 \\
\hline & 1.0 & 1.000000 & 1.000000 & 1.000000 \\
\hline \multirow{5}{*}{0.2} & 0.2 & 0.294598 & 1.129184 & 0.745861 \\
\hline & 0.4 & 0.565861 & 1.125361 & 0.765228 \\
\hline & 0.6 & 0.790149 & 1.111356 & 0.805133 \\
\hline & 0.8 & 0.943226 & 1.075366 & 0.877404 \\
\hline & 1.0 & 1.000000 & 1.000000 & 1.000000 \\
\hline \multirow{5}{*}{1.0} & 0.2 & 0.289501 & 1.117375 & 0.770576 \\
\hline & 0.4 & 0.558054 & 1.114159 & 0.789455 \\
\hline & 0.6 & 0.783347 & 1.102369 & 0.826209 \\
\hline & 0.8 & 0.940354 & 1.070841 & 0.890198 \\
\hline & 1.0 & 1.000000 & 1.000000 & 1.000000 \\
\hline \multirow{5}{*}{2.0} & 0.2 & 0.283965 & 1.106033 & 0.794738 \\
\hline & 0.4 & 0.549507 & 1.103393 & 0.813080 \\
\hline & 0.6 & 0.775806 & 1.093732 & 0.846574 \\
\hline & 0.8 & 0.937116 & 1.066580 & 0.902329 \\
\hline & 1.0 & 1.000000 & 1.000000 & 1.000000 \\
\hline
\end{tabular}

cross flow trend is observed at the central portion. Further, from Figure 8 it is remarked that, the temperature field diminished for the increasing values of $\beta$. Also, from Figure 9 it is observed that, the concentration profile increased for the increasing values of Casson fluid parameter in the flow region.

Figures 10-13 illustrates the effect of Hartmann number $(\mathrm{Ha})$ on velocity, temperature and concentration profiles in the flow region, respectively. Figure 10 portrays that, the radial velocity profile suppressed for the enhancing values of $H a$. This fact is mainly due to the reason that, an increment in magnetic field leads to the increase of Lorentz forces related to the magnetic field and these increased Lorentz forces offers the more resistance to the velocity field. Therefore, it is obvious to expect that, the velocity field will suppress for the increasing values of Hartmann number in the flow region. Further, from Figure 11 it is noticed that, the axial velocity profile diminished in the region $0 \leq \eta \leq 0.48$ and it is raised in the other portion of the channel for the increasing values of $H a$. Due to this variation in axial velocity, there occurs cross flow behaviour at the central portion of the channel. Further, from Figure 12 it is noticed that, the temperature profile increased for the increasing values of $\mathrm{Ha}$. This variation in temperature profile is mainly due to the reason that, the presence of Joule heating effect causes the thickening of temperature boundary layer, so that, the thermal field enhanced with increasing values of $\mathrm{Ha}$. Consequently, higher values of $\mathrm{Ha}$ are preferred for the case where the heating is required and vice-versa, the smaller 
Table 3: Numerical values of $F(\eta), \theta(\eta)$ and $\phi(\eta)$ for different set of $S$, obtained based on bvp4c scheme with fixed values of $\beta=0.7, H a=$ $0.1, \Omega_{T}=0.5, \Omega_{C}=1.5, N_{t}=N_{b}=0.1, E c=0.1, P r=0.7, K r=0.5$

\begin{tabular}{|c|c|c|c|c|}
\hline \multicolumn{5}{|c|}{ Results obtained based on bvp $4 c$ technique } \\
\hline$S$ & $\eta$ & $F(\eta)$ & $\theta(\eta)$ & $\phi(\eta)$ \\
\hline \multirow{5}{*}{-0.6} & 0.2 & 0.300431 & 1.144459 & 0.714475 \\
\hline & 0.4 & 0.574736 & 1.139843 & 0.734390 \\
\hline & 0.6 & 0.797793 & 1.122993 & 0.778066 \\
\hline & 0.8 & 0.946403 & 1.081340 & 0.860671 \\
\hline & 1.0 & 1.000000 & 1.000000 & 1.000000 \\
\hline \multirow{5}{*}{-0.4} & 0.2 & 0.298894 & 1.140249 & 0.723064 \\
\hline & 0.4 & 0.572404 & 1.135851 & 0.742836 \\
\hline & 0.6 & 0.795793 & 1.119775 & 0.785501 \\
\hline & 0.8 & 0.945575 & 1.079683 & 0.865309 \\
\hline & 1.0 & 1.000000 & 1.000000 & 1.000000 \\
\hline \multirow{5}{*}{-0.2} & 0.2 & 0.297412 & 1.136313 & 0.731137 \\
\hline & 0.4 & 0.570150 & 1.132120 & 0.750769 \\
\hline & 0.6 & 0.793854 & 1.116787 & 0.792471 \\
\hline & 0.8 & 0.944771 & 1.078141 & 0.869614 \\
\hline & 1.0 & 1.000000 & 1.000000 & 1.000000 \\
\hline \multirow{5}{*}{0.2} & 0.2 & 0.294597 & 1.129185 & 0.745861 \\
\hline & 0.4 & 0.565861 & 1.125361 & 0.765228 \\
\hline & 0.6 & 0.790149 & 1.111360 & 0.805129 \\
\hline & 0.8 & 0.943226 & 1.075367 & 0.877403 \\
\hline & 1.0 & 1.000000 & 1.000000 & 1.000000 \\
\hline \multirow{5}{*}{1.0} & 0.2 & 0.289502 & 1.117374 & 0.770577 \\
\hline & 0.4 & 0.558054 & 1.114159 & 0.789457 \\
\hline & 0.6 & 0.783347 & 1.102368 & 0.826211 \\
\hline & 0.8 & 0.940354 & 1.070840 & 0.890200 \\
\hline & 1.0 & 1.000000 & 1.000000 & 1.000000 \\
\hline \multirow{5}{*}{2.0} & 0.2 & 0.283965 & 1.106041 & 0.794727 \\
\hline & 0.4 & 0.549508 & 1.103401 & 0.813069 \\
\hline & 0.6 & 0.775807 & 1.093740 & 0.846564 \\
\hline & 0.8 & 0.937117 & 1.066586 & 0.902319 \\
\hline & 1.0 & 1.000000 & 1.000000 & 1.000000 \\
\hline
\end{tabular}

values of $\mathrm{Ha}$ are chosen for the case where the cooling is necessary. Also, it is observed from Figure 13 that, the concentration distribution is diminished for the magnifying values of $\mathrm{Ha}$ in the flow region.

The effect of thermal relaxation time parameter is described via Cattaneo-Christov heat and mass flux models using the coupled flow equations. Certainly, flow behaviour influences the heat and mass transfer process. However, at the same time the temperature field significantly influences the concentration distribution in the flow region. This is due to the presence of Brownian motion and thermophoresis effects in the temperature and concentration equations. Further, these effects are negligible on velocity field due to small temperature changes does not affect the viscosity of the fluid. But these effects are considerably noticed on concentration distribution.

Thus, the influence of $\Omega_{T}$ on thermal and concentration profiles is portrayed in the Figures 14 and 15. Figure 14 describes that, the temperature field suppressed for the magnifying values of $\Omega_{T}$. This variation in temperature profile is mainly due to the fact that, when the values of $\Omega_{T}$ raises, then the nano particles requires extra additional time to transfer the heat energy to their adjacent particles, this situation describe the non-conducting behaviour of the material medium and causes to decay the temperature profile in flow regime. However, the case $\Omega_{T}=0$ corresponds to the flow of heat with infinite speed and hence, temperature field is pre-dominant when $\Omega_{T}=0$. Thus, it is observed that, the temperature profile suppressed in 


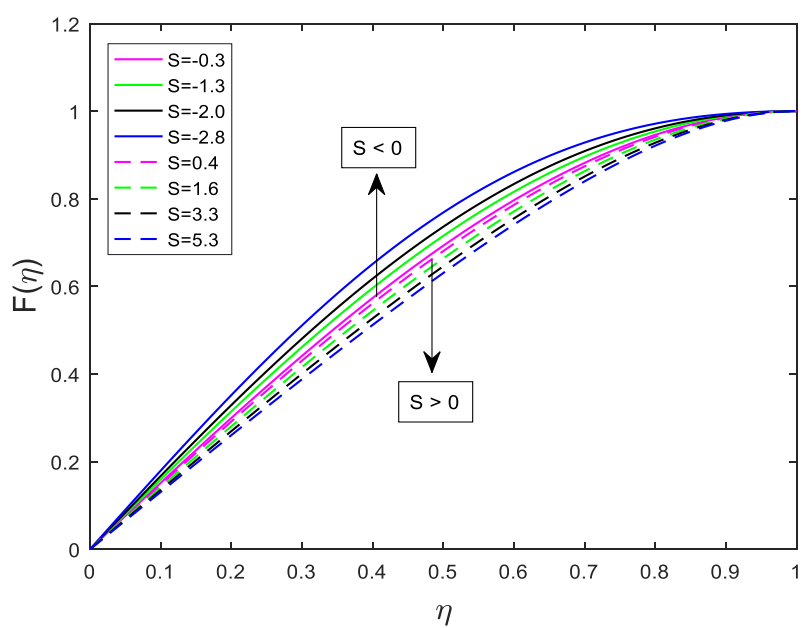

Figure 2: Effect of $S$ on $F(\eta)$ for fixed $\beta=2.2, H a=\Omega_{T}=N_{t}=$ $0.1, \Omega_{C}=E c=0.5, \operatorname{Pr}=S c=0.7$, and $K r=N_{b}=0.5$

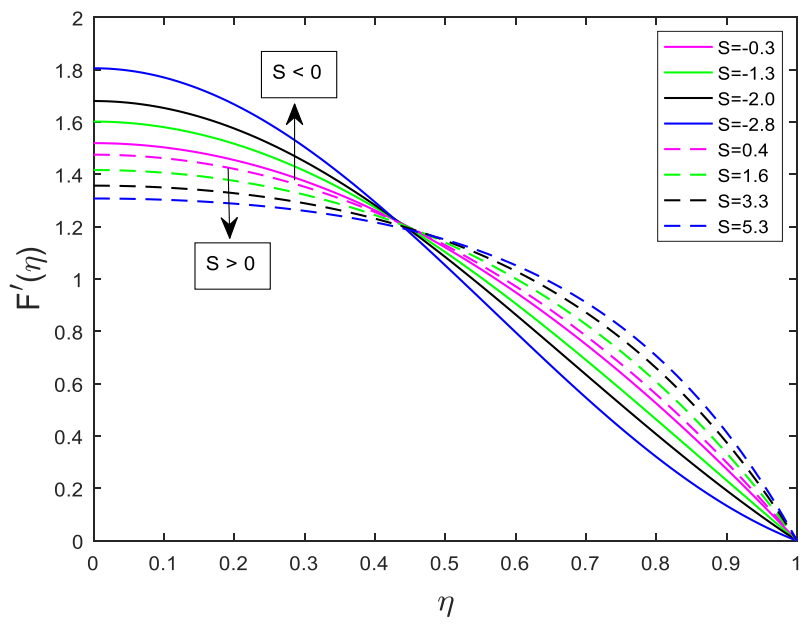

Figure 3: Effect of $S$ on $F^{\prime}(\eta)$ for fixed $\beta=2.2, H a=\Omega_{T}=N_{t}=$ $0.1, \Omega_{C}=E c=0.5, P r=S c=0.7$, and $K r=N_{b}=0.5$

case of Cattaneo-Christov heat flux model when compared to classical Fourier's law of heat conduction. Further, it is noticed from Figure 15 that, the concentration profile increased for the increasing values of $\Omega_{T}$ in the flow regime.

The influence of thermophoresis and Brownian motion forces characterizes the nanoparticles volume fraction diffusion and that correlates with one another in the neighborhood of boundaries which in turn considerably influence the concentration relaxation time parameter in the flow region. However, with the coupled energy and concentration equations, it is noticed that, the $\Omega_{C}$ significantly effects the thermal and concentration profiles. Further, influence of $\Omega_{C}$ on velocity field is comparatively neg-

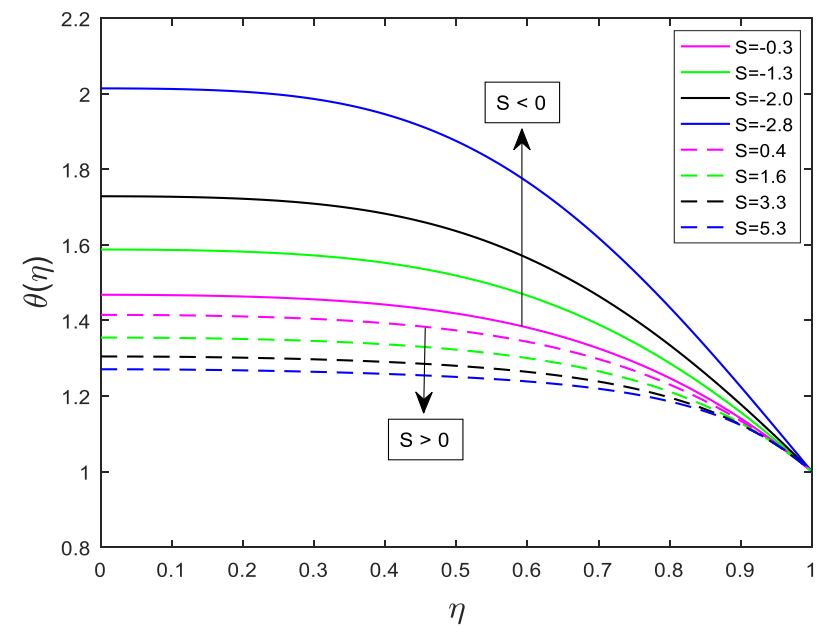

Figure 4: Effect of $S$ on $\theta(\eta)$ for fixed $\beta=2.2, H a=\Omega_{T}=N_{t}=$ $0.1, \Omega_{C}=E c=0.5, \operatorname{Pr}=S c=0.7$, and $K r=N_{b}=0.5$

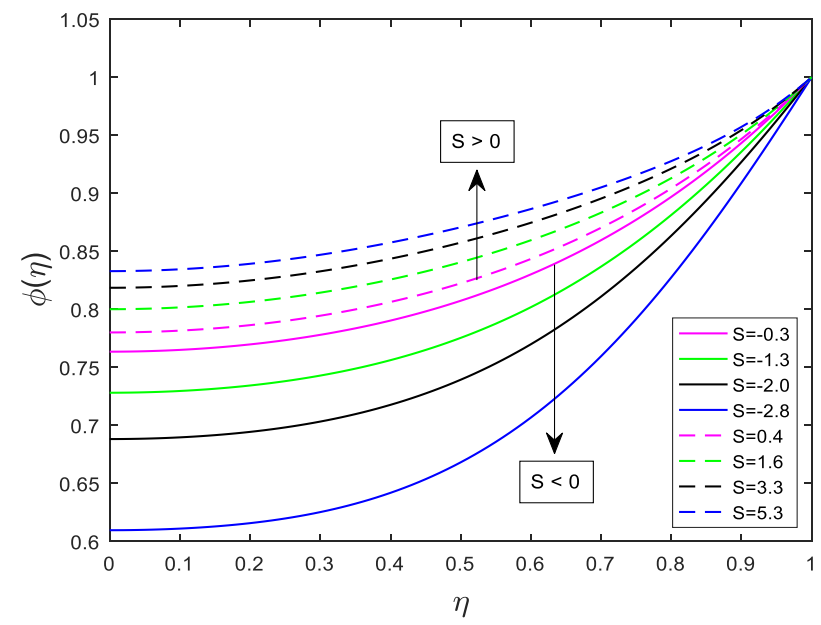

Figure 5: Effect of $S$ on $\phi(\eta)$ for fixed $\beta=2.2, H a=\Omega_{T}=N_{t}=$ $0.1, \Omega_{C}=E c=0.5, \operatorname{Pr}=S c=0.7$, and $K r=N_{b}=0.5$

ligible. However, this situation well described in the Figures 16 and 17. It is observed from Figure 16 that, the temperature field diminished with increase of $\Omega_{C}$. Further it is remarked from Figure 17 that, the concentration profile suppressed for the increasing values of $\Omega_{C}$. The physical behaviour noticed in Figure 17 is justified as follows, when $\Omega_{C}$ increases, the nanofluid particles need more time to diffuse through the material medium and causes to decay the concentration profile. However, for $\Omega_{C}=0$, diffusion process occurs at an infinite speed. Further, the concentration field dominates in case of $\Omega_{C}=0$. Thus, it is observed that, the concentration field suppressed in case of Cattaneo-Christov mass flux model when compared to Fick's law. 


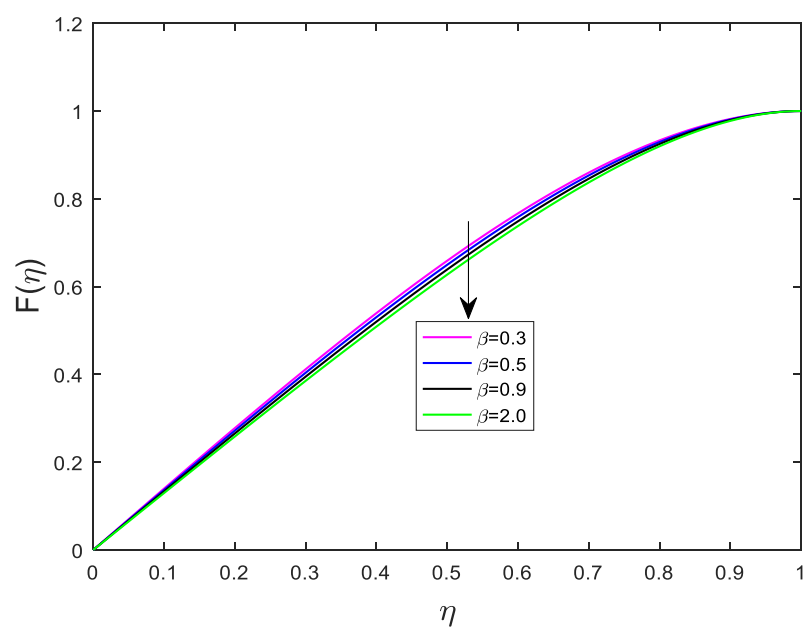

Figure 6: Effect of $\beta$ on $F(\eta)$ for fixed $S=6.0, H a=\Omega_{C}=N_{t}=K r=$ $0.1, \Omega_{T}=E c=0.5, N_{b}=0.3, P r=1.5$ and $S c=0.7$

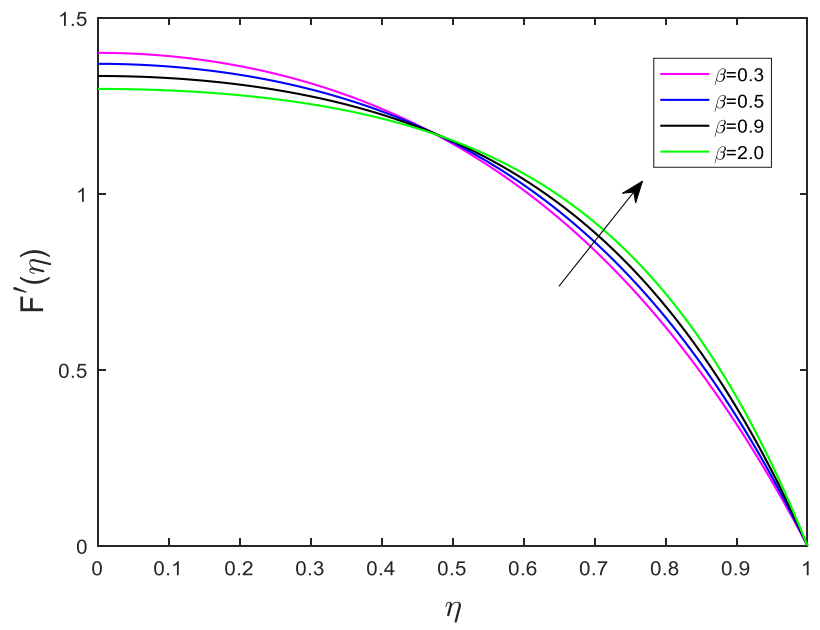

Figure 7: Effect of $\beta$ on $F^{\prime}(\eta)$ for fixed $S=6.0, H a=\Omega_{C}=N_{t}=K r=$ $0.1, \Omega_{T}=E c=0.5, N_{b}=0.3, P r=1.5$ and $S c=0.7$

Further, the presence of Brownian motion and thermophoresis parameters in temperature and concentration equations, significantly affects the thermal and concentration fields. Thus, the influence of thermophoresis parameter $\left(N_{t}\right)$ on temperature and concentration fields is portrayed in the Figures 18 and 19. However, it is remarked from Figure 18 that, the temperature profile increases with the increasing values of $N_{t}$. This increment in thermal profile is mainly due to the strong thermophoresis forces in flow region and hence these forces cause the increase in temperature distribution in the flow regime. Further, from Figure 19 it is observed that, the nanoparticle concentration profile diminished for the increasing values of thermophoresis parameter in the flow region.

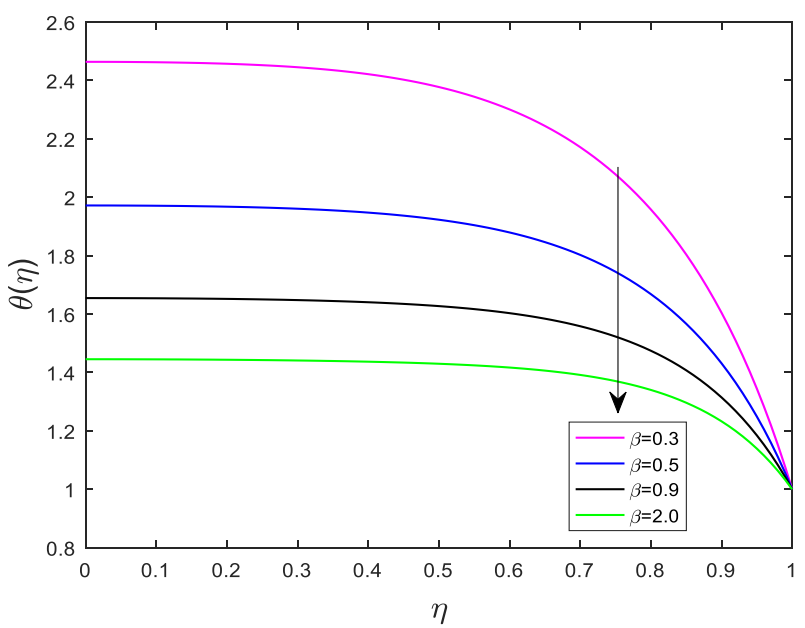

Figure 8: Effect of $\beta$ on $\theta(\eta)$ for fixed $S=6.0, H a=\Omega_{C}=N_{t}=K r=$ $0.1, \Omega_{T}=E c=0.5, N_{b}=0.3, P r=1.5$ and $S c=0.7$

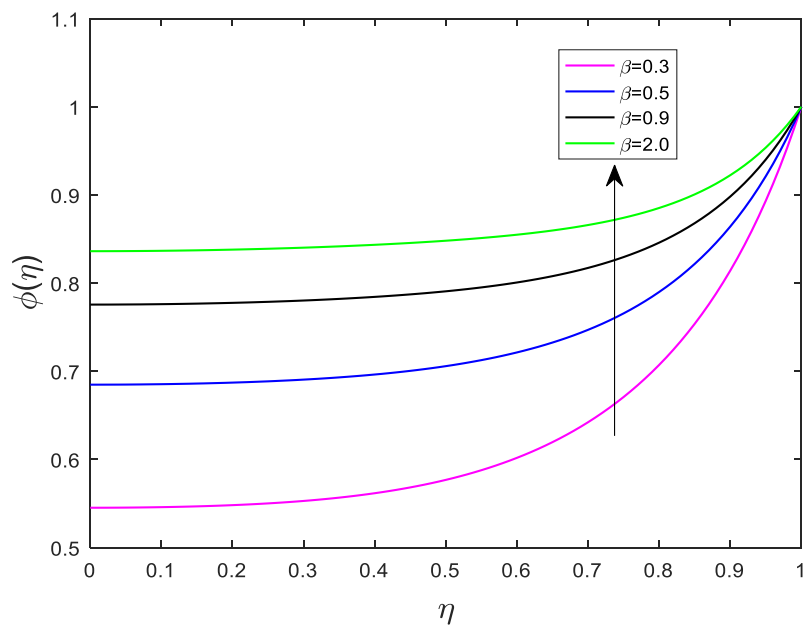

Figure 9: Effect of $\beta$ on $\phi(\eta)$ for fixed $S=6.0, \mathrm{Ha}=\Omega_{C}=N_{t}=\mathrm{Kr}=$ $0.1, \Omega_{T}=E c=0.5, N_{b}=0.3, \operatorname{Pr}=1.5$ and $S c=0.7$

Further, Figures 20 and 21 are presented to describe the effect of Brownian motion parameter $\left(N_{b}\right)$ on temperature and nanoparticle concentration distributions. However, it is noticed from Figure 20 that, the temperature profile suppressed for the enhancing values of $N_{b}$. Also, it is remarked from Figure 21 that, the nanoparticle concentration distribution enhanced for the increasing values of Brownian motion parameter $\left(N_{b}\right)$. This variation in concentration field is mainly due to the reason that, an increment in $N_{b}$ increases the Brownian motion diffusion coefficient and which makes the concentration field to enhance in flow region. Hence, increased concentration field is noticed for the enhanced Brownian motion parameter. Also, from Figure 21 it is noticed that, the effect of $N_{b}$ 


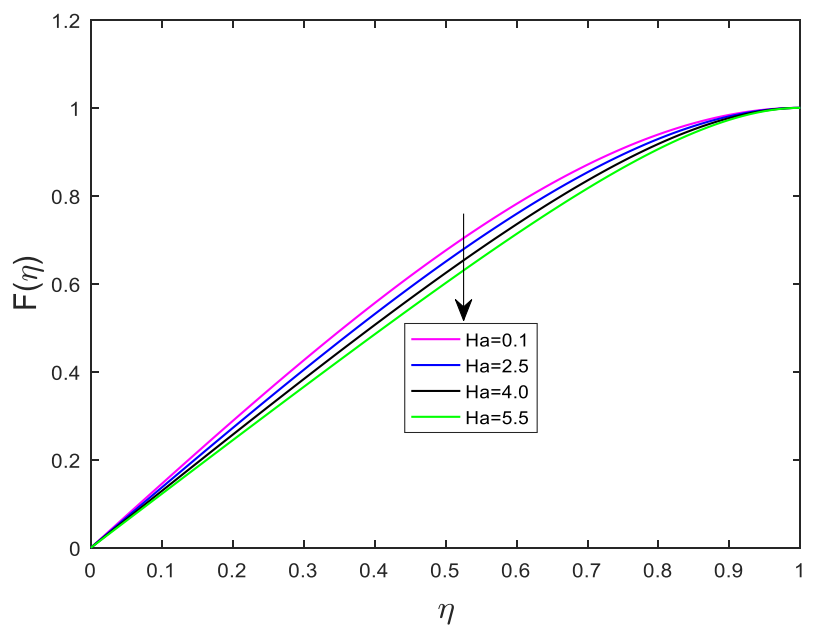

Figure 10: Effect of $\mathrm{Ha}$ on $\mathrm{F}(\eta)$ for fixed $S=0.6, \beta=5.0, N_{t}=$ $K r=0.1, \Omega_{T}=0.5, \Omega_{C}=1.5, E c=0.1, N_{b}=2.0, P r=$ 0.7 and $S c=0.7$

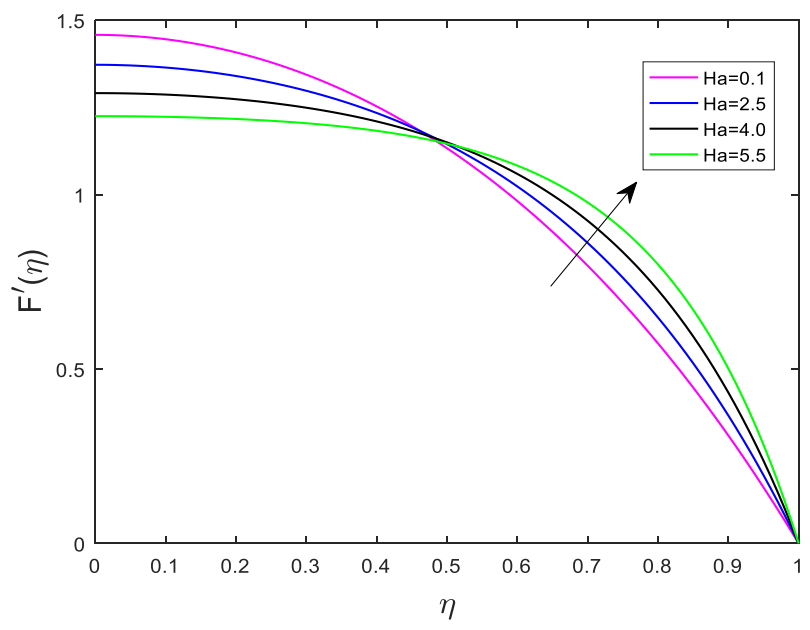

Figure 11: Effect of $\mathrm{Ha}$ on $F^{\prime}(\eta)$ for fixed $S=0.6, \beta=5.0, N_{t}=$ $K r=0.1, \Omega_{T}=0.5, \Omega_{C}=1.5, E c=0.1, N_{b}=2.0, P r=$ 0.7 and $S c=0.7$

on nanoparticles concentration is negligibly small beyond the value 1.0, but it is significant in the region $0 \leq \eta \leq 0.95$.

Further, the influence of Eckert number $(E c)$ on temperature and concentration profiles is illustrated in the Figures 22 and 23. It is observed from Figure 22 that, the temperature profile enhanced for the increasing values of Eckert number. However, this upsurge in temperature profile is expected because $E$ chas direct impact on the heat dissipation process and hence increases the temperature field in the flow region. Also, the thickness of thermal boundary layer diminished upon the increase of $E c$. It is

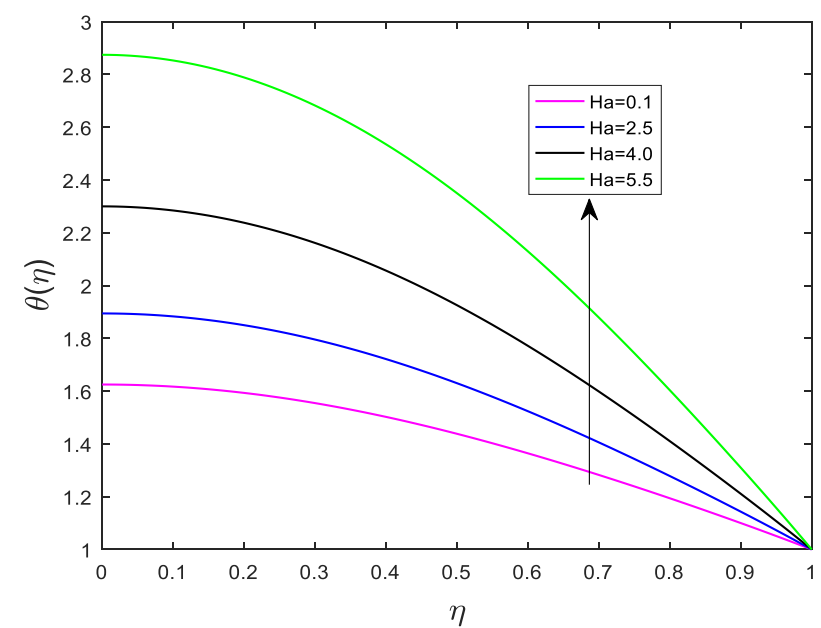

Figure 12: Effect of $\mathrm{Ha}$ on $\theta(\eta)$ for fixed $S=0.6, \beta=5.0, N_{t}=$ $K r=0.1, \Omega_{T}=0.5, \Omega_{C}=1.5, E c=0.1, N_{b}=2.0, P r=$ 0.7 and $S c=0.7$

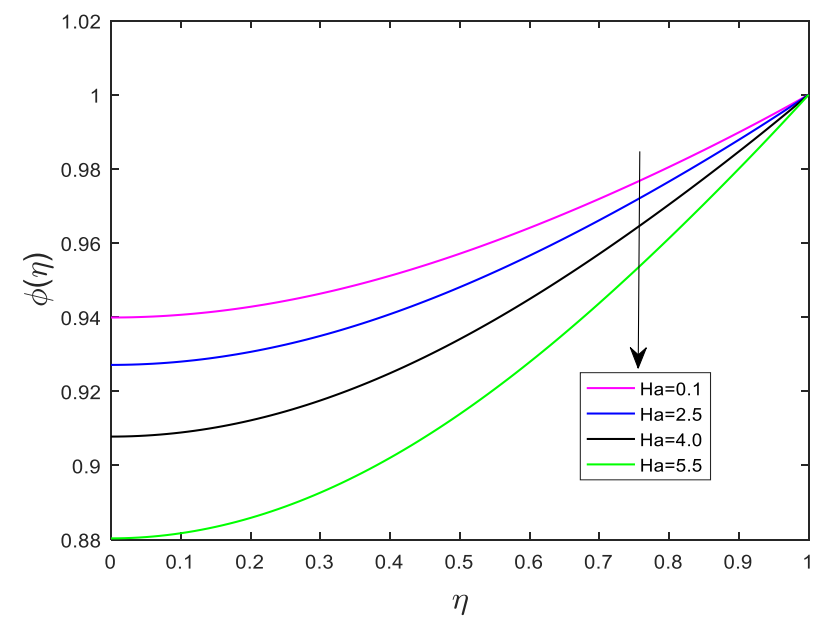

Figure 13: Effect of $\mathrm{Ha}$ on $\phi(\eta)$ for fixed $S=0.6, \beta=5.0, N_{t}=$ $K r=0.1, \Omega_{T}=0.5, \Omega_{C}=1.5, E c=0.1, N_{b}=2.0, P r=$ 0.7 and $S c=0.7$

quite obvious that, Eckert number is mainly used to determine the dissipated heat in the given medium/system. Further, from Figure 23 it is remarked that, the concentration profile diminished for the increasing values of Eckert number in the flow regime. This variation in concentration field is due to the presence of Brownian motion and thermophoresis effects in the energy and concentration equations.

Similarly, the impact of Prandtl number (Pr) on temperature and concentration profiles is depicted in the Figures 24 and 25. However, it is remarked from the Figure 24 that, the temperature profile profile enhanced for the in- 


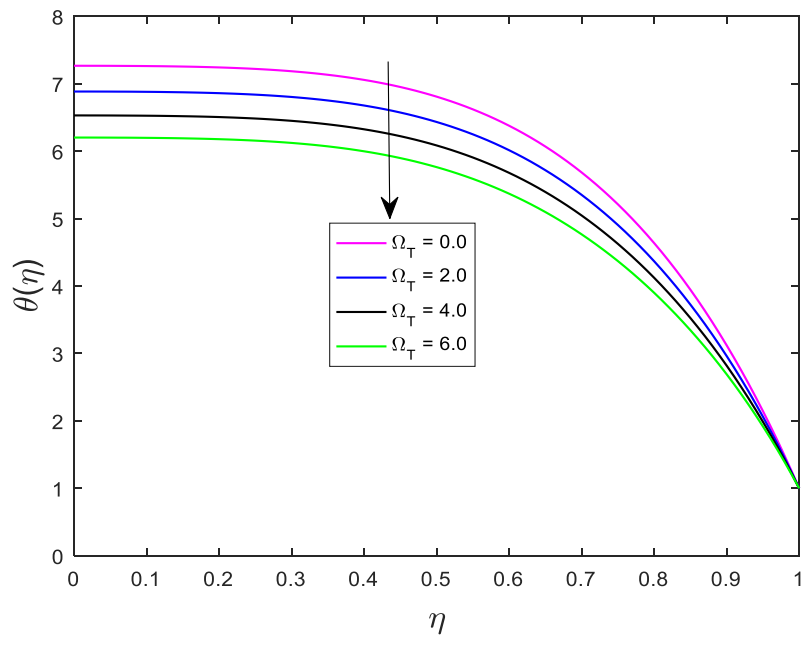

Figure 14: Effect of $\Omega_{T}$ on $\theta(\eta)$ for fixed $S=N_{b}=E c=0.5, \beta=$ $H a=N_{t}=\Omega_{C}=K r=0.1, P r=1.5$ and $S c=0.7$

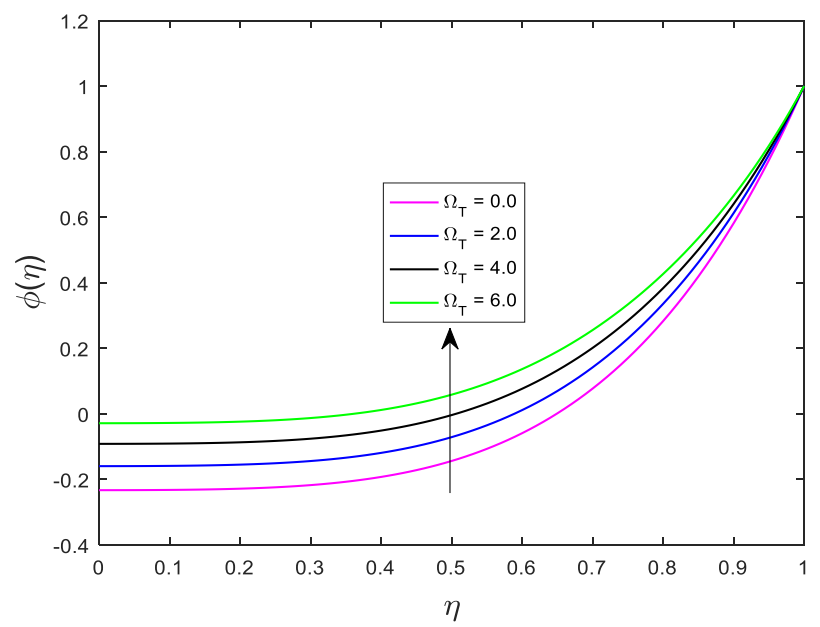

Figure 15: Effect of $\Omega_{T}$ on $\phi(\eta)$ for fixed $S=N_{b}=E c=0.5, \beta=$ $H a=N_{t}=\Omega_{C}=K r=0.1, P r=1.5$ and $S c=0.7$

creasing values of Prandtl number in the flow region. This variation in temperature profile is mainly due to the fact that, the larger values of $\operatorname{Pr}$ considerably reduces the thermal diffusivity and decreases the thickness of the thermal boundary layer and which in turn responsible for the increment of thermal profile in the flow regime. Further, it is expected that, the $\operatorname{Pr}<1$ related to physical objects with small viscosity \& larger thermal diffusivity. Also, $P r>1$ related to oils with smaller thermal diffusivity and high viscosity. Further, it is noticed from Figure 25 that, the concentration profile decreased for the increasing values of $P r$. This effect is observed due the coupled effects of Brownian motion and thermophoresis parameters in temperature and concentration equations.

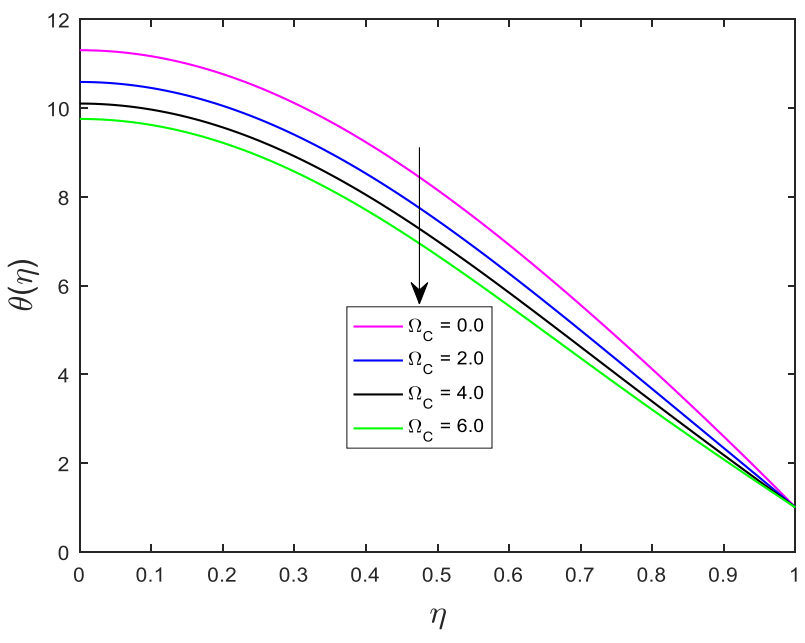

Figure 16: Effect of $\Omega_{C}$ on $\theta(\eta)$ for fixed $S=1.5, \Omega_{T}=N_{b}=$ $0.5, \beta=H a=0.1, N_{t}=-0.1, E c=0.1, P r=0.7, K r=$ -0.5 and $S c=0.7$

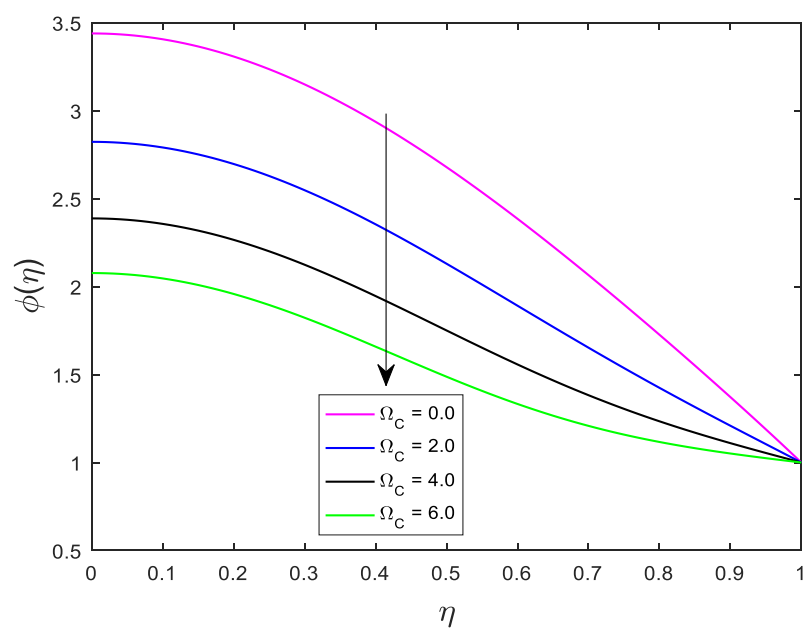

Figure 17: Effect of $\Omega_{C}$ on $\phi(\eta)$ for fixed $S=1.5, \Omega_{T}=N_{b}=$ $0.5, \beta=H a=0.1, N_{t}=-0.1, E c=0.1, P r=0.7, K r=$ -0.5 and $S c=0.7$

Further, the effect of Schmidt number $(S c)$ on temperature and concentration profiles is illustrated in the Figures 26 and 27. Figure 26 describes that, the temperature profile decrease for the increasing values of Schmidt number in the flow region. However, it is clear from Figure 26 that, the Schmidt number has no considerable effect on temperature profile. Furthermore, the influence of Schmidt number on concentration profile is depicted in the Figure 27. However, it is remarked from Figure 27 that, the concentration field suppressed for the increasing values of Schmidt number. This reduction in concentration field is mainly due to the fact that, the larger values of 


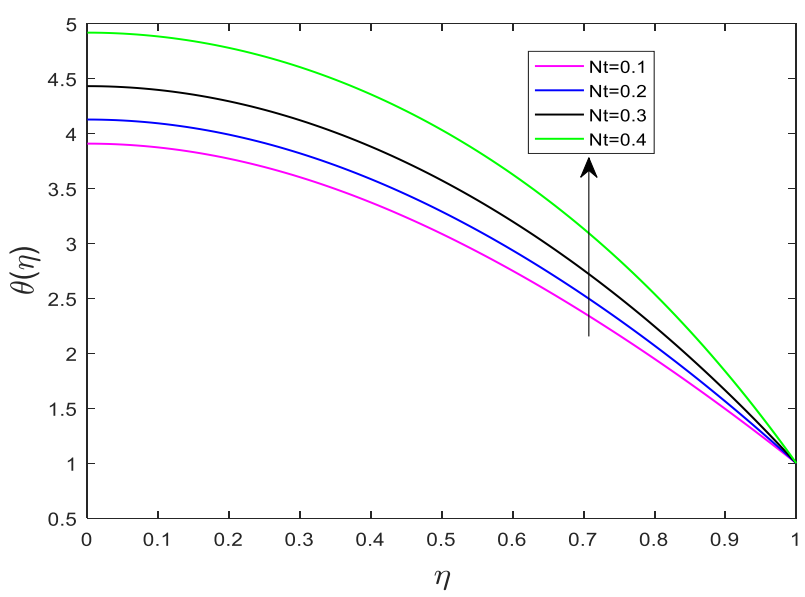

Figure 18: Effect of $N_{t}$ on $\theta(\eta)$ for fixed $S=H a=N_{b}=0.5, \beta=$ $\Omega_{T}=\Omega_{C}=0.1, E c=0.1, P r=0.7, K r=2.0$ and $S c=2.0$

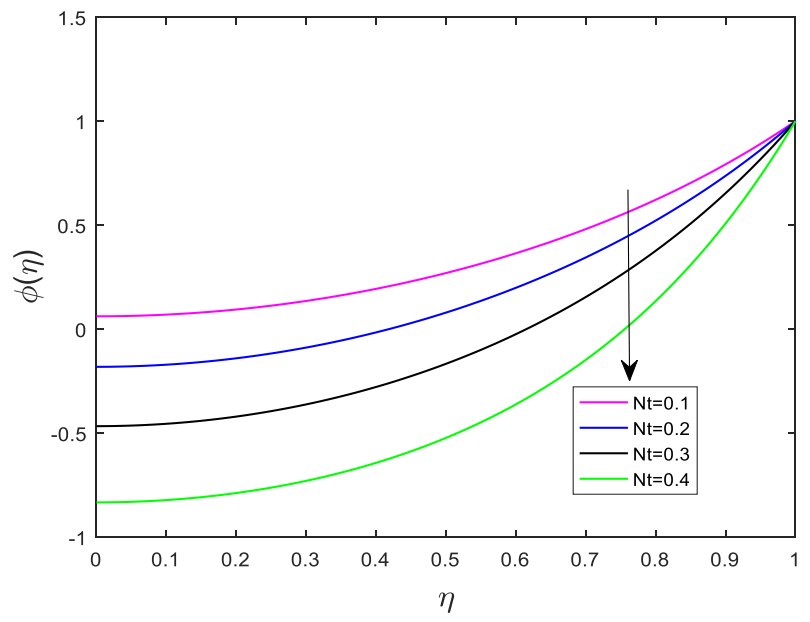

Figure 19: Effect of $N_{t}$ on $\phi(\eta)$ for fixed $S=H a=N_{b}=0.5, \beta=$ $\Omega_{T}=\Omega_{C}=0.1, E c=0.1, \operatorname{Pr}=0.7, K r=2.0$ and $S c=2.0$

$S c$ decreases the mass diffusivity and hence concentration profile decreases.

Due to the presence of thermophoresis and Brownian motion effects in the energy and concentration equations, the time-dependent chemical reaction parameter influences the both temperature and concentration fields in the flow region significantly. Thus, this effect was clearly shown in the Figures 28 and 29, respectively. It is noticed from Figure 28 that, the temperature profile diminished for the destructive chemical reaction $(K r>0)$ and it is enhanced for the constructive chemical reaction $(\mathrm{Kr}<0)$ in the flow regime. Further, from Figure 29 it is observed that, the concentration profile decreased for the destructive chemical reaction and it is increased for the generative chemical reaction.

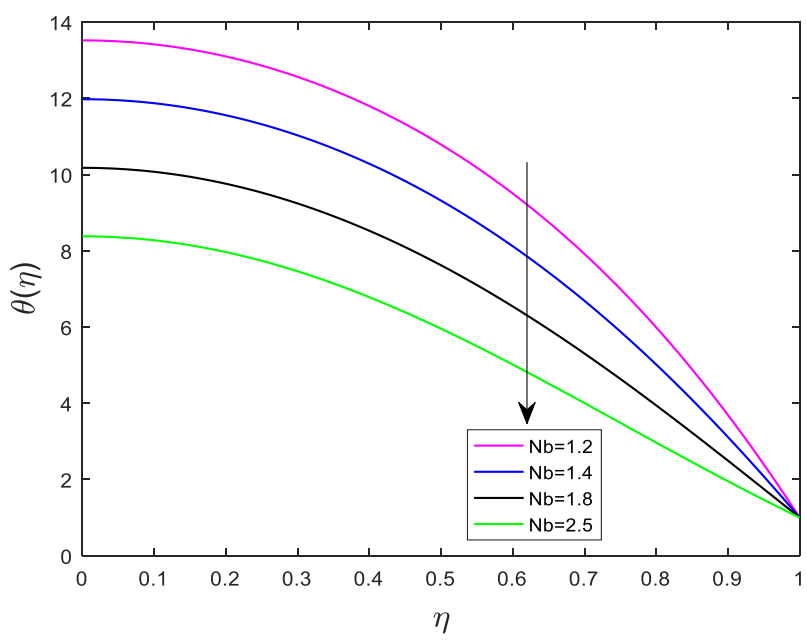

Figure 20: Effect of $N_{b}$ on $\theta(\eta)$ for fixed $S=1.5, \beta=$ $\Omega_{T}=\Omega_{C}=0.1, N_{t}=0.2, \mathrm{Ha}=0.8, E c=0.3, \mathrm{Pr}=0.7, \mathrm{Kr}=$ 2.0 and $S c=2.0$

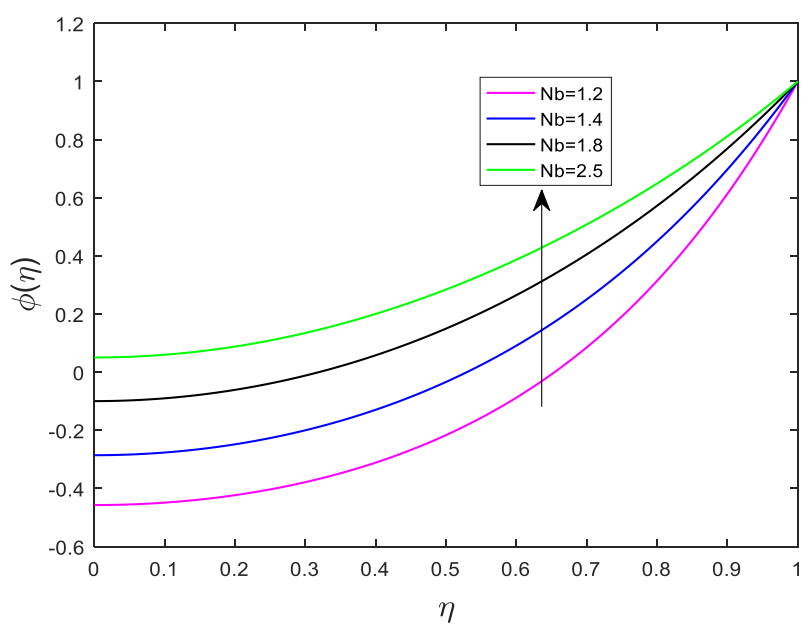

Figure 21: Effect of $N_{b}$ on $\phi(\eta)$ for fixed $S=1.5, \beta=$ $\Omega_{T}=\Omega_{C}=0.1, N_{t}=0.2, \mathrm{Ha}=0.8, E c=0.3, \mathrm{Pr}=0.7, \mathrm{Kr}=$ 2.0 and $S c=2.0$

However, from the industrial point of view, the quantities of engineering interest such as momentum, heat and mass transport coefficients have large number of advantageous. Also, the heat and mass transfer properties of viscous fluids are identified mainly in terms of skin-friction, heat and mass rates. Further, the numerical values of skinfriction coefficient $\left(F^{\prime \prime}(\eta)\right)$, heat $\left(\theta^{\prime}(\eta)\right)$ and mass transfer rates $\left(\phi^{\prime}(\eta)\right)$ at $\eta=1$ for different values of control parameters are calculated and tabulated in the Tables 4-6, respectively. From Table 4 it is noticed that, the magnitude of momentum transport coefficient enhanced for the increasing values of squeezing and Hartmann numbers. Further, the magnitude of skin-friction coefficient diminished for 


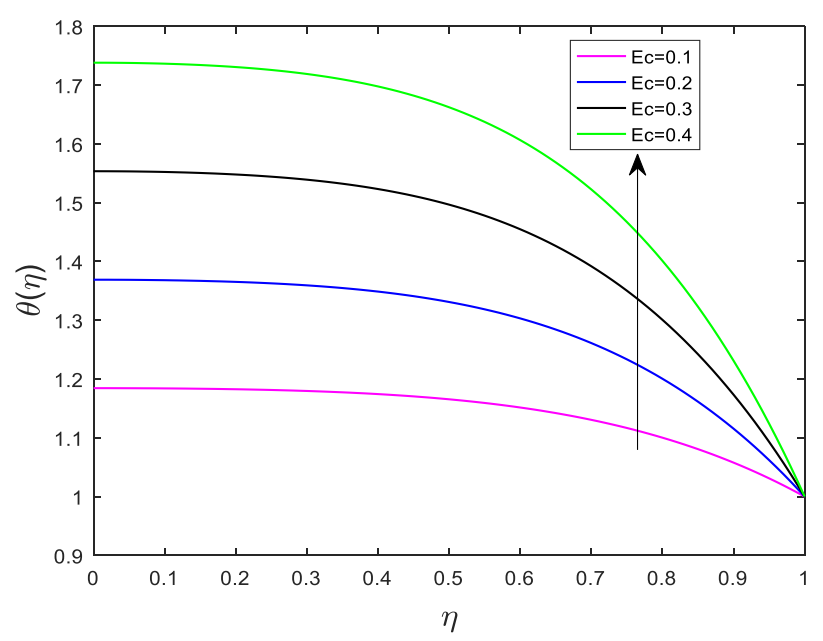

Figure 22: Effect of $E c$ on $\theta(\eta)$ for fixed $S=\Omega_{C}=N_{t}=E c=K r=$ $0.1, H a=0.2, N_{b}=\beta=\Omega_{T}=0.5, \operatorname{Pr}=0.7$, and $S c=0.7$

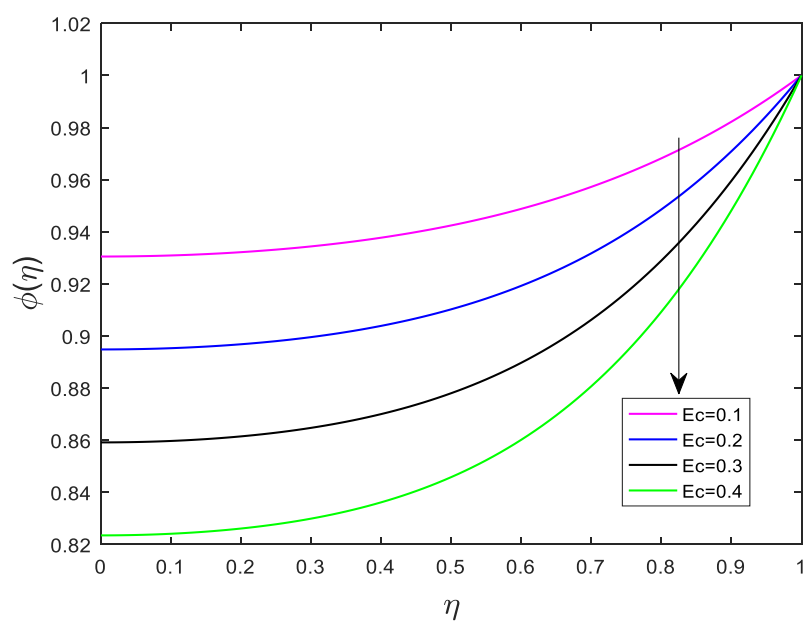

Figure 23: Effect of $E c$ on $\phi(\eta)$ for fixed $S=\Omega_{C}=N_{t}=E c=K r=$ $0.1, H a=0.2, N_{b}=\beta=\Omega_{T}=0.5, P r=0.7$, and $S c=0.7$

the increasing values of Casson fluid parameter in the flow regime.

Furthermore, the numerical values of heat transfer rate $\left(\theta^{\prime}(\eta)\right)$ for different values of physical parameters are tabulated in the Table 5 . It is clearly noticed from Table 5 that, the magnitude of Nusselt number suppressed for the increasing values of Brownian motion and thermal relaxation time parameters. Further, it is noticed that, the magnitude of Nusselt number amplified for the increasing values of thermophoresis and concentration relaxation time parameters.

Similarly, the behaviour of Sherwood number for various values of control parameters is illustrated in the Table 6. It is remarked from Table 6 that, the magnitude of mass transfer rate diminished for the increasing values of Brow-

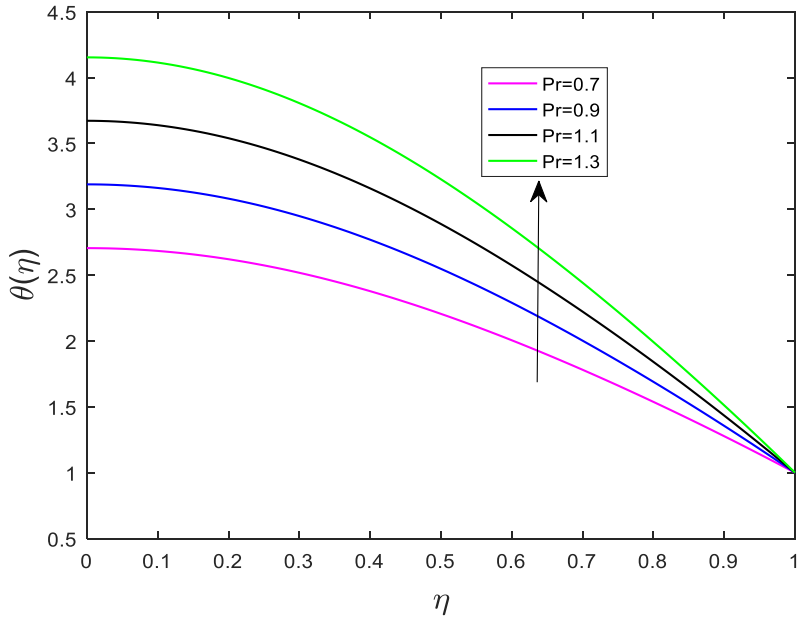

Figure 24: Effect of $\operatorname{Pr}$ on $\theta(\eta)$ for fixed $S=N_{t}=E c=K r=$ $0.1, H a=N_{b}=\beta=\Omega_{T}=\Omega_{C}=0.5$ and $S c=0.7$

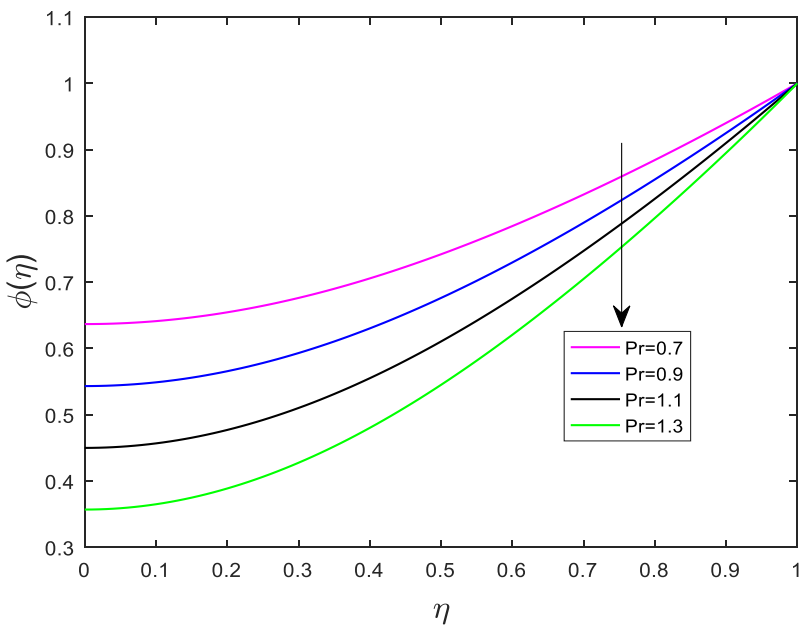

Figure 25: Effect of $\operatorname{Pr}$ on $\phi(\eta)$ for fixed $S=N_{t}=E c=K r=$ $0.1, H a=N_{b}=\beta=\Omega_{T}=\Omega_{C}=0.5$ and $S c=0.7$

nian motion and concentration relaxation time parameters. Also, it is observed that, the magnitude of Sherwood number enhanced for the magnifying values of Schmidt number and time-dependent chemical reaction parameter in the flow region.

\section{Conclusions}

The present numerical investigation address the combined effects of heat and mass transfer in squeezing flow of magneto-hydrodynamic, unsteady Casson nanofluid flow between parallel plates by considering the generalized Cattaneo-Christov heat and mass flux models with tran- 
Table 4: Numerical values of skin-friction coefficient for various values of $S, \beta, \mathrm{Ha}$ with fixed $\Omega_{T}=\Omega_{C}=0.5, N_{b}=N_{t}=0.1, E c=$ $0.5, \mathrm{Kr}=0.1, \mathrm{Pr}=0.7$ and $S c=0.7$

\begin{tabular}{cccc}
\hline$S$ & $\beta$ & $H a$ & $-\left(1+\frac{1}{\beta}\right) F^{\prime \prime}(1)$ \\
\hline-1.2 & 2.0 & 0.2 & 3.54752565 \\
-0.8 & & 3.89393676 \\
-0.4 & & 4.21251044 \\
0.4 & & 4.78403205 \\
1.0 & & 5.16770925 \\
2.0 & & 5.74292647 \\
1.5 & 0.4 & & 11.52481345 \\
& 0.8 & & 7.74807668 \\
& 1.5 & & 5.97395156 \\
& 2.0 & & 5.46417599 \\
& 3.5 & 0.1 & 4.80571173 \\
& 4.0 & 0.4 & 4.69554490 \\
& & 0.8 & 5.45923830 \\
& & 1.4 & 5.48388496 \\
& & & 5.56206233 \\
& & & 5.77184566 \\
& & & 6.08224120 \\
\hline
\end{tabular}

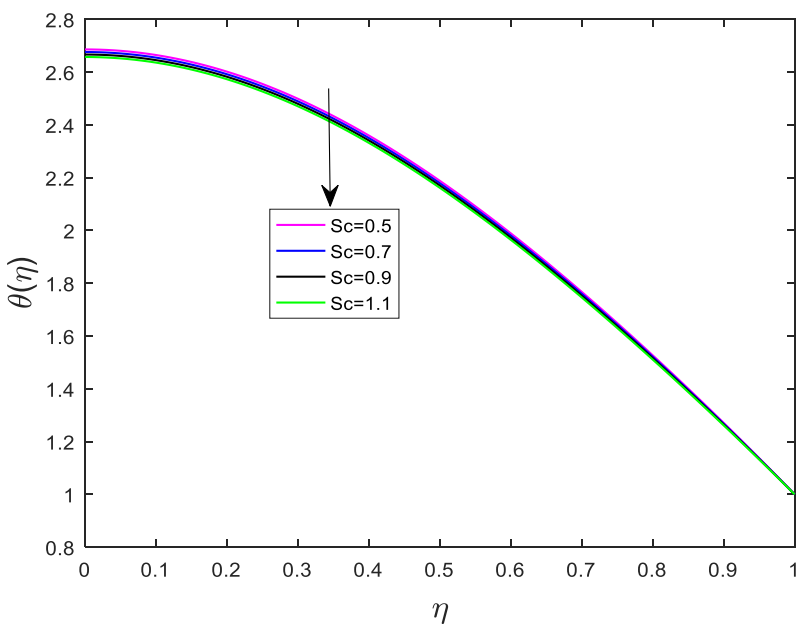

Figure 26: Effect of $S c$ on $\theta(\eta)$ for fixed $S=N_{t}=E c=K r=$ $0.1, H a=N_{b}=\beta=\Omega_{T}=\Omega_{C}=0.5$ and $\operatorname{Pr}=0.7$

sient chemical reaction process. Due to the moment of upper plate with velocity $\left(\frac{-\alpha l}{2 \sqrt{1-\alpha t}}\right)$ towards or away from the lower plate, the motion of the fluid is produced. The timedependent, coupled highly nonlinear PDEs governing the present physical problem are solved by utilizing RK-SM and bvp4c methods. The physical significance of the considered problem is described by portraying the behaviour of the different control parameters on flow, temperature and concentration distributions. Also, the obtained numerical data for various values of control parameters are expressed in terms of graphs and tables. Thus, the following are the major finding of the present numerical study.

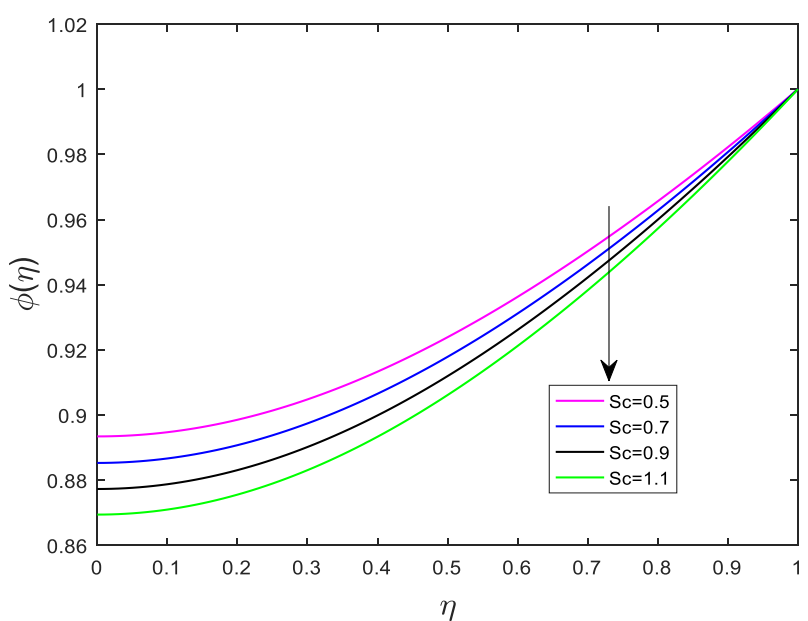

Figure 27: Effect of $S c$ on $\phi(\eta)$ for fixed $S=N_{t}=E c=K r=$ $0.1, H a=N_{b}=\beta=\Omega_{T}=\Omega_{C}=0.5$ and $P r=0.7$

- Temperature field is diminished in Cattaneo-Christov thermal diffusion model when compared to classical Fourier's law.

- Concentration field is suppressed in CattaneoChristov mass diffusion model when compared to conventional Fick's law.

- Temperature field is an increasing function of thermophoresis parameter.

- The influence of $S>0$ and $S<0$ on axial flow is opposite.

- Magnifying $\mathrm{Ha}$ diminish the radial and axial velocity field. 
Table 5: Numerical values of heat transfer rate for various values of $\Omega_{T}, \Omega_{C}, N_{t}$ and $N_{b}$ with fixed $S=0.4, \beta=1.4, \mathrm{Ha}=0.1, \mathrm{Pr}=$ $0.7, E c=0.1, S c=0.7$ and $\mathrm{Kr}=0.1$

\begin{tabular}{lllll}
\hline$\Omega_{T}$ & $\Omega_{C}$ & $N_{t}$ & $N_{b}$ & $-\theta^{\prime}(1)$ \\
\hline 0.5 & 0.1 & 0.1 & 0.36152971 \\
1.0 & & & 0.35925278 \\
2.0 & & & 0.35476328 \\
3.0 & & & 0.35035857 \\
4.0 & & & 0.34603742 \\
5.0 & & & 0.34179862 \\
0.1 & & & 0.36338074 \\
& 0.5 & & 0.36339798 \\
& 1.0 & & 0.36343195 \\
& 2.0 & & 0.36346527 \\
& 3.0 & & 0.36349793 \\
& 4.0 & & 0.36342121 \\
& & & 0.36347560 \\
& 0.2 & & 0.36358445 \\
& 0.5 & & 0.36369339 \\
& 0.7 & & 0.36380242 \\
& 0.9 & & 0.36289701 \\
& & & 0.36242807 \\
& & & 0.36149286 \\
& & & 0.36056118 \\
& & 0.3 & 0.35963302 \\
\hline
\end{tabular}

Table 6: Numerical values of mass transfer rate for various values of $N_{b}, \Omega_{C}, S c, K r$ with fixed $\Omega_{T}=0.4, \beta=0.5, S=H a=0.5, N_{t}=$ $0.1, E c=0.5, P r=0.7$

\begin{tabular}{ccccc}
\hline$N_{b}$ & $\Omega_{C}$ & $S c$ & $K r$ & $-\phi^{\prime}(1)$ \\
\hline 0.2 & 0.5 & 0.7 & 0.1 & 1.63811787 \\
0.3 & & & 1.11252710 \\
0.5 & & & 0.69205936 \\
0.7 & & & 0.51186354 \\
0.9 & & & 0.41175834 \\
1.0 & & & 0.37672273 \\
0.5 & & & 0.69331776 \\
& 0.4 & & 0.68582173 \\
& 1.0 & & 0.67361497 \\
& 2.0 & & 0.66175944 \\
& 3.0 & & & 0.65024700 \\
& 4.00 & & & 0.70830525 \\
& & & 0.75607156 \\
& & & 0.79561081 \\
& & & 0.82859057 \\
& & & 0.85629528 \\
& & & -0.59217504 \\
& & & -0.05920362 \\
& & & 0.97447178 \\
& & & -1.5 & 1.05651901 \\
& & & 0.7 & 1.13371927 \\
\hline
\end{tabular}




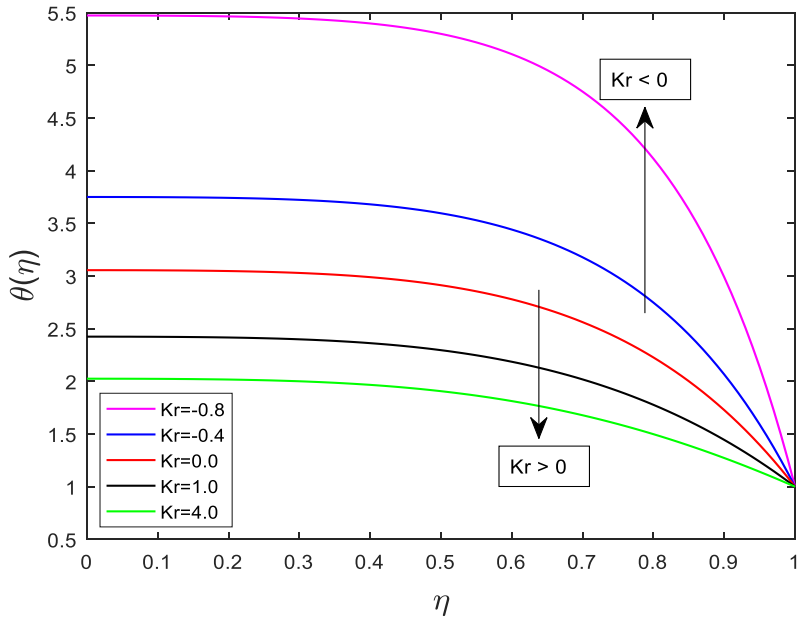

Figure 28: Effect of $K r$ on $\theta(\eta)$ for fixed $S=S c=1.0, N_{b}=$ 5.0, $E c=\beta=0.5, H a=N_{t}=\Omega_{T}=\Omega_{C}=0.1, K r=-0.8$ and $\mathrm{Pr}=$ 2.0

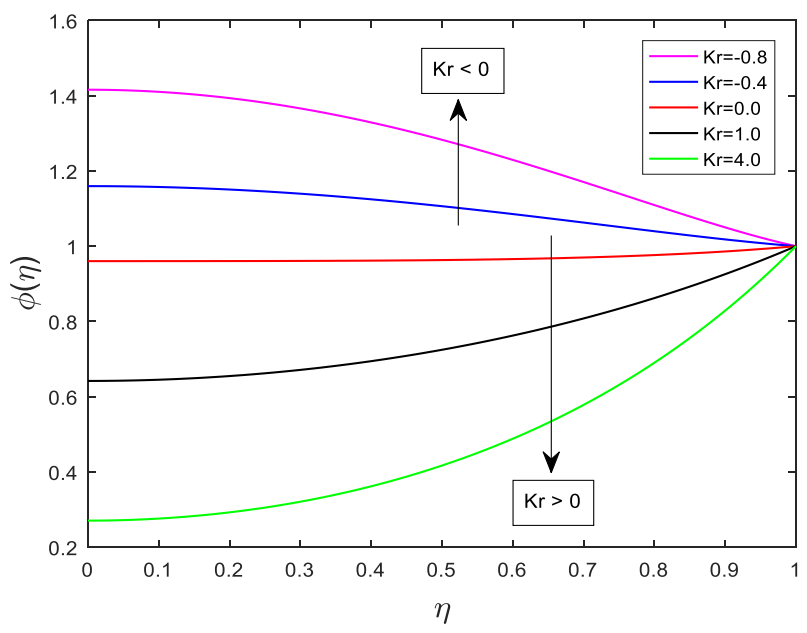

Figure 29: Effect of $K r$ on $\phi(\eta)$ for fixed $S=S c=1.0, N_{b}=$ 5.0, $E c=\beta=0.5, H a=N_{t}=\Omega_{T}=\Omega_{C}=0.1, K r=-0.8$ and $\mathrm{Pr}=$ 2.0

- Temperature field is a diminishing function of Brownian motion parameter.

- Effects of $P r$ and $E c$ on temperature and concentration fields are found to be similar.

- Concentration field is enhanced for the constructive chemical reaction and decreased for the destructive chemical reaction.

- Magnifying Schmidt number suppress the concentration distribution.

- Skin-friction coefficient decreases with increase in $S$ and $\mathrm{Ha}$.

- Nusselt number increases with increase in $N_{b}$ and $\Omega_{T}$.

- Sherwood number diminished with increment in $K r$.

\section{Future research work}

However, it is anticipated that, the present paper gives a motivation for the mathematical modelling of squeezing problems and advantageous in bearings, motors and lubrication, heating/cooling processes, injection modeling, compression, moisture migration, food and polymer processing, viscometers, rheometer and in elongation flows etc. Further, the present investigation may be helpful to analyze the squeezing flow occurring in the bioengineering and biology. For example, the valves, diarthrodial joints and blood flow problems. Also, the present results may be extended to analyze the characteristics of time-dependent retardation or relaxation phenomenon in non-Newtonian fluids. Also, the squeezing flow of shear thinning/thickening fluids like third-grade, second-grade fluids may be demonstrated.

Acknowledgements: The authors wish to express their gratitude to the reviewers who highlighted important areas for improvement in this earlier draft of the article. Their suggestions have served specifically to enhance the clarity and depth of the interpretation of results in the revised manuscript. One of the author Usha Shankar wishes to thank Karnataka Power Corporation limited, Raichur Thermal Power Station, Shaktinagar, for their encouragement.

\section{Nomenclature}

$S \quad$ Squeezing number

$\mathrm{Ha} \quad$ Hartmann number

$\Omega_{T} \quad$ Thermal relaxation time parameter

$\Omega_{C} \quad$ Concentration relaxation time parameter

$N_{b} \quad$ Brownian motion parameter

$N_{t} \quad$ Thermophoresis parameter

$\mathrm{Pr} \quad$ Prandtl number

Ec Eckert number

Sc Schmidt number

$\mathrm{Kr}$ Time-dependent chemical reaction parameter

$\kappa \quad$ Thermal conductivity

$D_{B} \quad$ Coefficient of Brownian motion

$D_{T} \quad$ Coefficient of thermophoresis

$C_{f} \quad$ Skin-friction coefficient

$\mathrm{Nu} \quad$ Nusselt number

$\mathrm{Sh} \quad$ Sherwood number

$B_{0} \quad$ Uniform magnetic field

$T$
Fluid temperature

Upper plate temperature

Fluid concentration

Upper plate concentration 
$k_{1}$

$u, v$

directions

$F^{\prime}, F \quad$ Dimensionless axial and radial velocities

\section{Greek symbols}

$\beta \quad$ Casson fluid parameter

$\sigma \quad$ Electrical conductivity

$\eta \quad$ Similarity variable

$\theta \quad$ Dimensionless temperature

$\phi \quad$ Dimensionless concentration

$\rho \quad$ Fluid density

$\mu \quad$ Dynamic viscosity

$\alpha \quad$ Squeezing rate

$v \quad$ Kinematic viscosity of the fluid

\section{References}

[1] Salata O.V., Applications of nanoparticles in biology and medicine, J. Nanobiotechnology, 2004, 2(3), 1-6.

[2] Giersig M., Khomutov G.B., Nanomaterials for application in medicine and biology, Springer, Dordrecht, Netherlands, 2008.

[3] Jong W.H.D., Borm P.J.A., Drug delivery and nanoparticles: Applications and hazards, Int. J. Nanomed., 2008, 3(2), 133149.

[4] Kolesnikov V.I., Myasnikova N.A., Volnyanko E.N., Ermakov S.F., Sychev A.P., Sychev A.A., Lubricants with ceramic nanoadditives and wear-resistant surface structures of heavyduty frictional joints, Russ. Eng. Res., 2011, 31(5), 454-457.

[5] Greco A., Mistry K., Sista V., Eryilmaz O., Erdemir A., Friction and wear behaviour of boron based surface treatment and nano-particle lubricant additives for wind turbine gearbox applications, Wear, 2011, 271, 1754-1760.

[6] Kurahatti R.V., Surendranathan A.O., Kori S.A., Singh N., Kumar A.V.R., Srivastava S., Defence applications of polymer nanocomposites, Defence Sci. J., 2010, 60(5), 551-563.

[7] Choi S.U.S., Eastman J.A., Enhancing thermal conductivity of fluids with nanoparticle, ASME International Mechanical Engineering Congress and Exposition, San Francisco, California, 1995, 231, 99-105.

[8] Engmann J., Servais C., Burbidge A.S., Squeeze flow theory and applications to rheometry: A review, J. Non-Newtonian Fluid Mech., 2005, 132, 1-27.

[9] Stefan M.J., Versuchüber die scheinbare adhesion, SitzungsberSächsAkadWissWein, Math-Nat Wiss Kl, 1874, 69, 713-721.

[10] Reynolds O., On the theory of lubrication, Transaction of Royal Society London, 1886, 177, 157-234.

[11] Archibald F.R., Load capacity and time relations for squeeze films, J. Lubr. Technol., 1956, 78, 231-245.

[12] Leider P.J., Bird R.B., Squeezing flow between parallel disks. I. Theoretical analysis, Ind. Eng. Chem., 1974, 13(4), 336-341.

[13] Domairry G., Aziz A., Approximate analysis of MHD squeeze flow between two parallel disks with suction or injection by homotopy perturbation method, Math. Prob. Eng., 2009, 2009, 1-19.

[14] Siddiqui A.M., Irum S., Ansari A.R., Unsteady squeezing flow of a viscous MHD fluid between parallel plates, a solution using the homotopy perturbation method, Math. Model. Anal., 2008, 13(4), 565-576.

[15] Rashidi M.M., Shahmohamadi H., Dinarvand S., Analytic approximate solutions for unsteady two dimensional and axisymmetric squeezing flows between parallel plates, Math. Prob. Eng., 2008, 2008, 1-13.

[16] Kaushik P., Mondal P.K., Chakraborty S., Flow dynamics of a viscoelastic fluid squeezed and extruded between two parallel plates, J. Non-Newton. Fluid Mech., 2016, 227, 56-64.

[17] Muhammad T., Hayat T., Alsaedi A., Qayyum A., Hydromagnetic unsteady squeezing flow of Jeffrey fluid between two parallel plates, Chin. J. Phys., 2017, 55(4), 1511-1522.

[18] Joneidi A.A., Domairry G., Babaelahi M., Effect of mass transfer on a flow in the magnetohydrodynamic squeeze film between two parallel disks with one porous disk, Chem. Eng. Commun., 2011, 198, 299-311.

[19] Hayat T., Yousaf A., Mustafa M., Obaidat S., MHD squeezing flow of second-grade fluid between two parallel disks, Int. J. Numer. Meth. Fluids., 2012, 69, 399-410.

[20] Duwairi H.M., Tashtoush B., Damseh R.A., On heat transfer effects of a viscous fluid squeezed and extruded between two parallel plates, Heat Mass Transfer, 2004, 41, 112-117.

[21] Mustafa M., Hayat T., Obaidat S., On heat and mass transfer in the unsteady squeezing flow between parallel plates, Meccanica, 2012, 47, 1581-1589.

[22] Khan H., Qayyum M., Khan O., Ali M., Unsteady squeezing flow of Casson fluid with magnetohydrodynamic effect and passing through porous medium, Math. Prob. Eng., 2016, 2016, 1-14.

[23] Khan U., Ahmed N., Zaidi Z.A., Asadullah M., Mohyud-Din S.T., MHD squeezing flow between two infinite plates, Ain Shams Eng. J., 2014, 5, 187-192.

[24] Dogonchi A.S., Ganji D.D., Study of nanofluid flow and heat transfer between non-parallel stretching walls considering Brownian motion, J. Taiwan Inst. Chem. Eng., 2016, 69, 1-13.

[25] Dogonchi A.S., Ganji D.D., Analytical Solution and heat transfer of two-phase nanofluid flow between non-parallel walls considering Joule heating effect, Powder Technol., 2017, 318, 390-400.

[26] Dogonchi A.S., Divsalar K., Ganji D.D., Flow and heat transfer of MHD nanofluid between parallel plates in the presence of thermal radiation, Comput. Methods Appl. Mech. Engrg., 2016, 310, 58-76.

[27] Sheikholeslami M., Ganji D.D., Nanofluid flow and heat transfer between parallel plates considering Brownian motion using DTM, Comput. Methods Appl. Mech. Engrg., 2015, 283, 651-663.

[28] Kandelousi M.S., KKL correlation for simulation of nanofluid flow and heat transfer in a permeable channel, Phys. Lett. A, 2014, 378(45), 3331-3339.

[29] Gerdroodbary M.B., Takami M.R., Ganji D.D., Investigation of thermal radiation on traditional Jeffery-Hamel flow to stretchable convergent/divergent channels, Case Stud. Therm. Eng., 2015, 6, 28-39.

[30] Mohammadian E., Ghasemi S.E., Poorgashti H., Hosseini M., Ganji D.D., Thermal investigation of Cu-water nanofluid between two vertical planes, Proc IMechE Part E: J Process Me- 
chanical Engineering, 2014, 229(1), 36-43.

[31] Pourmehran O., Gorji M.R., Bandpy M.G., Ganji D.D., Analytical investigation of squeezing unsteady nanofluid flow between parallel plates by LSM and CM, Alex. Eng J., 2015, 54, 17-26.

[32] Sheikholeslami M., Akbar N.S., Mustafa M.T., MHD effects on nanofluid with energy and hydrothermal behaviour between two collateral plates application of new semi analytical technique, Therm. Sci., 2017, 21(5), 2081-2093.

[33] Sheikholeslami M., Hatami M., Domairry G., Numerical simulation of two phase unsteady nanofluid flow and heat transfer between parallel plates in presence of time dependent magnetic field, J. Taiwan Inst. Chem. Eng., 2015, 46, 43-50.

[34] Fourier J.B.J., Theorie analytique De La Chaleur, Cambridge University Press, UK, 2009.

[35] Cattaneo C., Sulla conduzionedelcalore, Atti del Seminario Maermatico e Fisico, dell Universita di Modena e Reggio Emilia, 1948, 3, 83-101.

[36] Christov C.I., On frame indifferent formulation of the MaxwellCattaneo model of finite-speed heat conduction, Mech. Res. Commun., 2009, 36, 481-486.

[37] Ciarletta M., Straughan B., Uniqueness and structural stability for the Cattaneo-Christov equations, Mech. Res. Commun., 2010, 37, 445-447.

[38] Straughan B., Thermal convection with the Cattaneo-Christov model, Int. J. Heat Mass Transfer., 2010, 53, 95-98.

[39] Hayat T., Farooq M., Alsaedi A., Al-Solamy F., Impact of Cattaneo-Christov heat flux in the flow over a stretching sheet with variable thickness, AIP Adv., 2015, 5, 087159-12.

[40] Hayat T., Imtiaz M., Alsaedi A., Almezal S., On CattaneoChristov heat flux in MHD flow of Oldroyd-B fluid with homogeneous-heterogeneous reactions, J. Magn. Magn. Mater., 2016, 401, 296-303.

[41] Khan J.A., Mustafa M., Hayat T., Alsaedi A., Numerical study of Cattaneo-Christov heat flux model for viscoelastic flow due to an exponentially stretching surface, PLoS One, 2015, 10(9), 1-10.

[42] Mustafa M., Cattaneo-Christov heat flux model for rotating flow and heat transfer of upper-convected Maxwell fluid, AIP Adv., 2015, 5, 047109-10.
[43] Malik R., Khan M., Shafiq A., Mushtaq M., and Hussain M., An analysis of Cattaneo-Christov double-diffusion model for Sisko fluid flow with velocity slip, Results Phys., 2017, 7, 1232-1237.

[44] Shahid A., Bhatti M.M., Be'g O.A., Kadir A., Numerical study of radiative Maxwell viscoelastic magnetized flow from a stretching permeable sheet with the Cattaneo-Christov heat flux model, Neural Comput \& Applic., 2017, 30(11), 3467-3478.

[45] Dogonchi A.S., Ganji D.D., Impact of Cattaneo-Christov heat flux on MHD nanofluid flow and heat transfer between parallel plates considering thermal radiation effect, J. Taiwan Inst. Chem. Eng., 2017, 80, 52-63.

[46] Farooq M., Ahmad S., Javed M., Anjum A., Analysis of Cattaneo-Christov heat and mass fluxes in the squeezed flow embedded in porous medium with variable mass diffusivity, Results Phys., 2017, 7, 3788-3796.

[47] Muhammad N., Nadeem S., Mustafa T., Squeezed flow of a nanofluid with Cattaneo-Christov heat and mass fluxes, Results Phys., 2017, 7, 862-869.

[48] Farooq M., Ahmad S., Javed M., Anjum A., Chemically reactive species in squeezed flow through modified Fourier's and Fick's laws, Eur. Phys. J. Plus., 2018, 133, 1-18.

[49] Akmal N., Sagheer M., Hussain S., Numerical study focusing on the entropy analysis of MHD squeezing flow of a nanofluid model using Cattaneo-Christov theory, AIP Adv., 2018, 8, 055201-17.

[50] Casson N., Rheology of dispersed system, Pergamon Press, Oxford, UK, 1959.

[51] Cebeci T., Bradshaw P., Physical and computational aspects of convective heat transfer, Springer-Verlag, New York, USA, 1984.

[52] Kiusalaas J., Numerical methods in engineering with matlab, Cambridge University Press, UK, 2005.

[53] Wang C.Y., The squeezing of fluid between two plates, J. Appl. Mech., 1976, 43(4), 579-583.

[54] Khan U., Ahmed N., Khan S.I., Zaidi Z.A., Jun Y.X., MohyudDin S.T., On unsteady two-dimensional and axisymmetric squeezing flow between parallel plates, Alex. Eng. J., 2014, 53, 463-468. 